\title{
IMPROVING POWER OUTPUT IN OLDER ADULTS UTILIZING PLYOMETRICS IN AN ALTERG TREADMILL
}

by

Tyler Dobbs

\author{
A thesis \\ submitted in partial fulfillment \\ of the requirements for the degree of \\ Master of Science in Kinesiology, Biophysical Studies \\ Boise State University
}

August 2016 
(C) 2016

Tyler Dobbs

ALL RIGHTS RESERVED 


\title{
BOISE STATE UNIVERSITY GRADUATE COLLEGE
}

\section{DEFENSE COMMITTEE AND FINAL READING APPROVALS}

\author{
of the thesis submitted by
}

Tyler Dobbs

Thesis Title: Improving Power Output in Older Adults Utilizing Plyometrics in an AlterG Treadmill

Date of Final Oral Examination: 17 June 2016

The following individuals read and discussed the thesis submitted by student Tyler Dobbs, and they evaluated his presentation and response to questions during the final oral examination. They found that the student passed the final oral examination.

Scott A. Conger, Ph.D.

Co-Chair, Supervisory Committee

Shawn R. Simonson, Ed.D.

Co-Chair, Supervisory Committee

Tyler N. Brown, Ph.D.

Member, Supervisory Committee

The final reading approval of the thesis was granted by Scott A. Conger, Ph.D., Co-Chair of the Supervisory Committee, and Shawn R. Simonson, Ed.D., Co-Chair of the Supervisory Committee. The thesis was approved for the Graduate College by Jodi Chilson, M.F.A., Coordinator of Theses and Dissertations. 


\section{DEDICATION}

To the most amazing woman I know, my mother, Vera. The sacrifices you have made in my life are immeasurable. I would be nowhere close to where I am today without your enduring love and support. Thank you for always being my source of encouragement and positivity whenever I want to give up. I love you. 


\section{ACKNOWLEDGEMENTS}

I would like to thank all of the participants of the study. You were all wonderful to work with and your hard work did not go unnoticed. I would also like to thank everyone that helped out with data collection. Cameron Needham, Cassidy Fike, and Chris Mecham, I would not have been able to accomplish this without your help. I would also like to thank the members of my committee. Thank you Dr. Tyler Brown for your willingness to assist me in this project in your already very busy first year. Thank you Dr. Shawn Simonson for helping me come up with this idea and get it off the ground and running. Finally, thank you to Dr. Scott Conger, I wouldn't have accomplished this task without your support. My 2 minute questions that nearly always led to 1 hour conversations may have been the most informative part of this entire project. Thank you for always taking time to help, even if you didn't have it. I sincerely appreciate all of the knowledge that you all possess and thank you all for passing some of that along to myself. 


\begin{abstract}
The purpose of this study was to compare functional strength and power output in older adults who completed plyometric training in an AlterG treadmill compared to older adults who completed traditional resistance training. Methods: Twenty-three older adults were randomized to a strength (SG), plyometric (PG), or control group (CG). SG and PG exercised 3x/week for 8 weeks while CG performed no exercise. Measures of a timed sitto-stand, stair climb, estimated maximal muscular strength, and isokinetic power were taken at pre and post testing. A repeated measures $2 \times 3$ (time $\mathrm{x}$ group) ANOVA was used to determine if there was a significant main effect. A one-way ANOVA was run on the differences in pre and post and post-hoc analysis with the Bonferroni adjustment using pairwise comparisons then determined differences. Results: PG was significantly faster than CG in the chair sit-to-stand $(\mathrm{p}=0.013)$, stair climb time $(\mathrm{p}=0.002)$, stair climb power output $(\mathrm{p}<0.001)$, leg extension $(\mathrm{p}=0.009)$, and lunge $(\mathrm{p}=0.03)$. PG was significantly more powerful at all 3 velocities in both flexion and extension compared to SG and CG ( $p<0.001)$ with the exception of $60^{\circ} / \mathrm{sec}$ extension. Conclusion: Eight weeks of plyometrics in an AlterG Treadmill can significantly improve performance of a chair sit-to-stand task, improve the time to climb a flight of stairs, significantly increase muscular strength in the leg press, leg extension, single leg lunge, and significantly increase isokinetic knee flexor and extensor power in older adults. In this study, the PG was able to increase muscular strength at the same rate or better than the SG without performing any resistance training. Also, the PG outperformed SG in functional tasks. To
\end{abstract}


the author's knowledge, this is the first plyometric training study focusing on older adults. Results suggest that plyometrics, if modified and performed in a safe environment, can increase muscular strength and power and improve functional abilities in older adults. 


\section{TABLE OF CONTENTS}

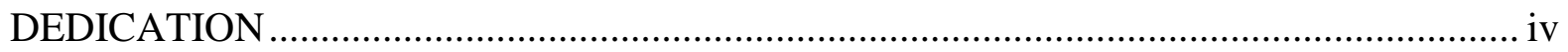

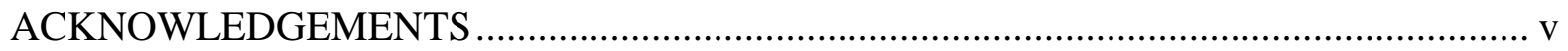

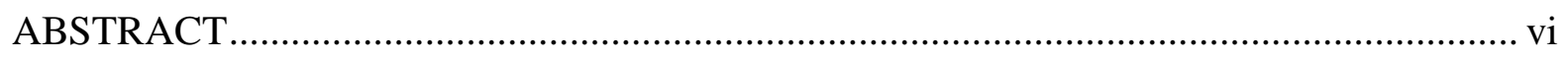

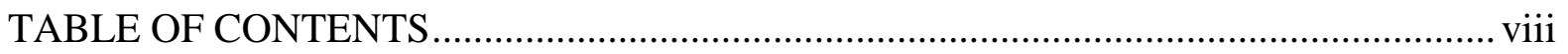

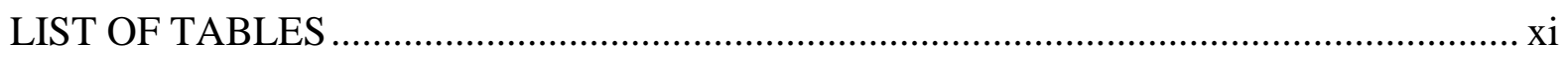

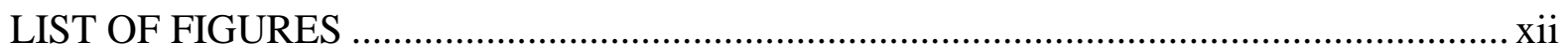

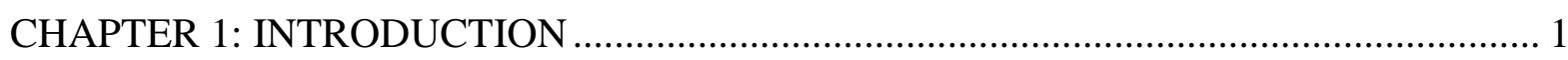

Purpose

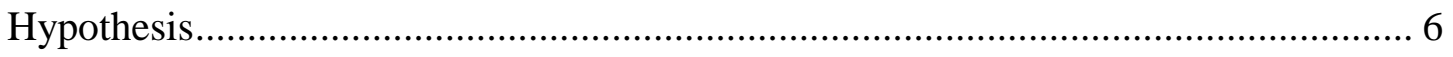

Significance

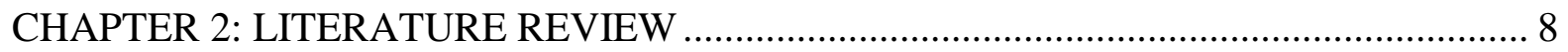

Muscular Strength Training in Older Adults ............................................................. 10

Muscular Power Training in Older Adults............................................................ 13

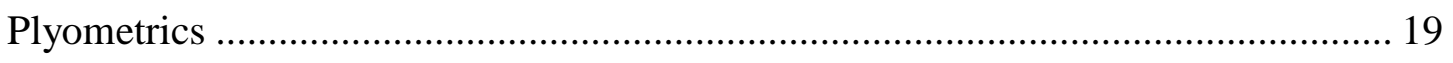

Anti-gravity Treadmills ...................................................................................... 23

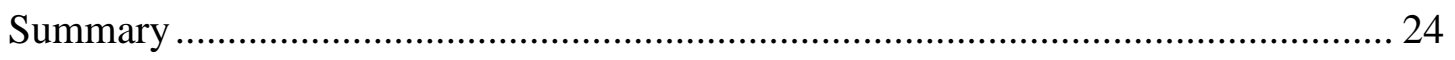

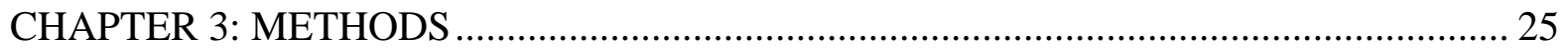

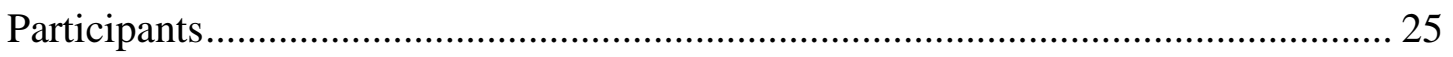

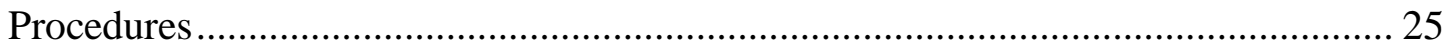

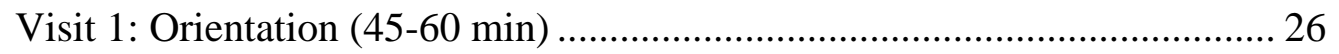


Visit 2: Pre-Tests (60-90 minutes) ........................................................ 26

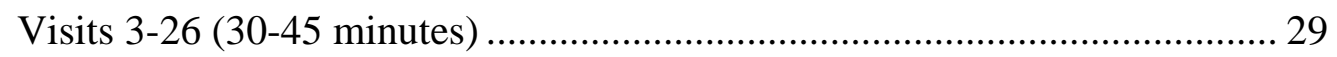

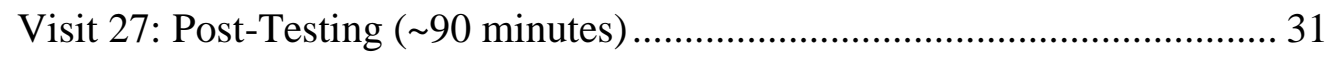

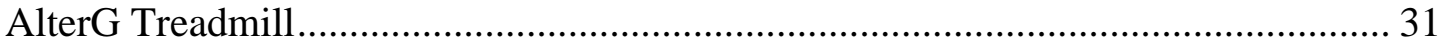

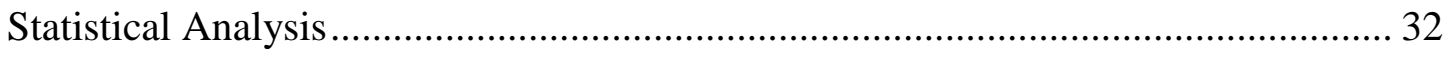

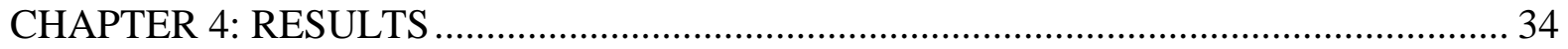

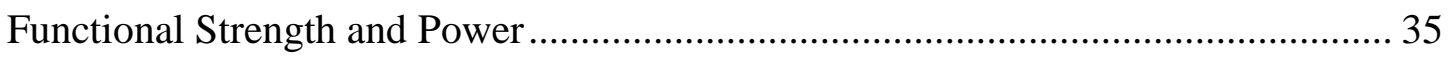

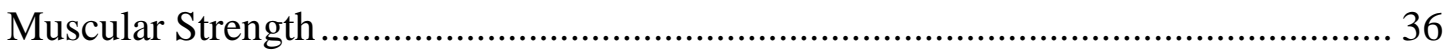

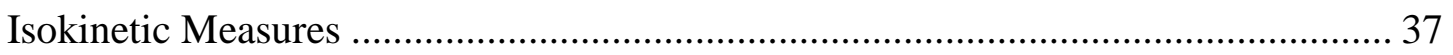

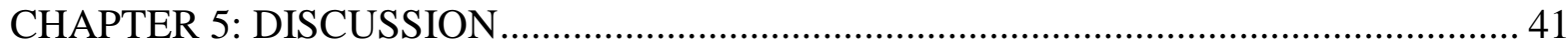

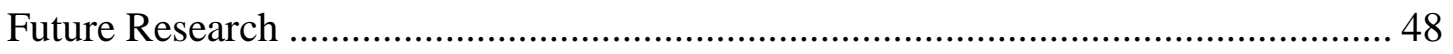

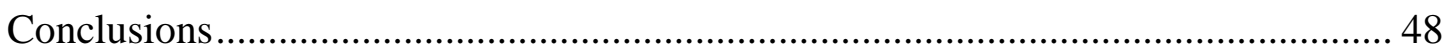

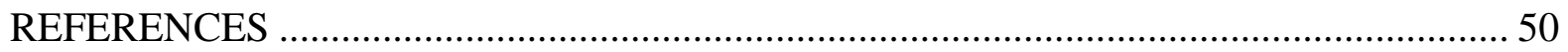

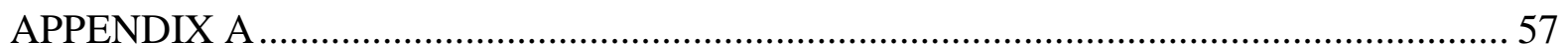

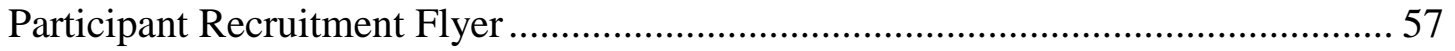

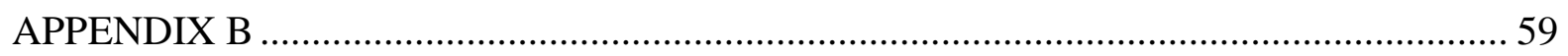

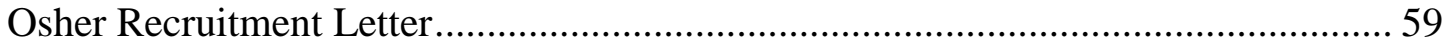

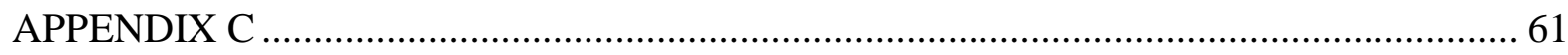

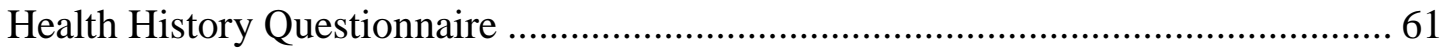

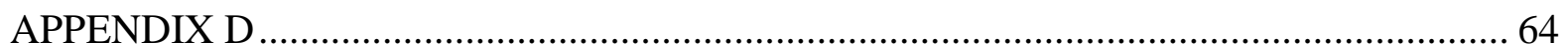

Boise State University Institutional Review Board Approval Letter......................... 64

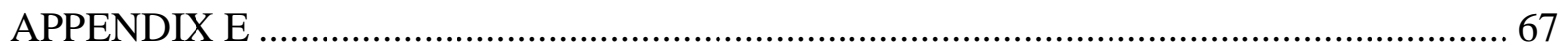

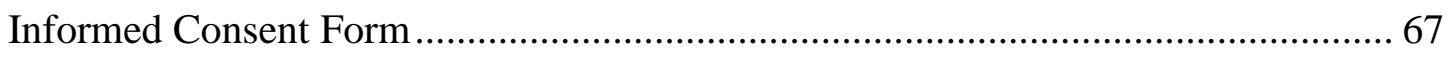




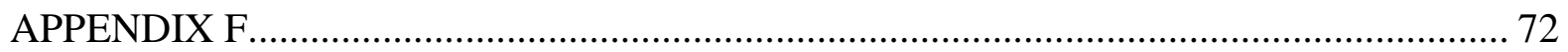

Physician Consent to Participate ...................................................................... 72

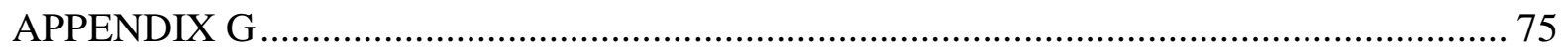

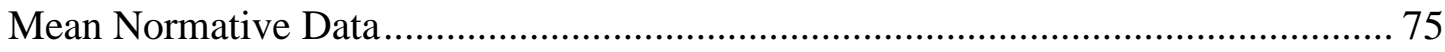

Strength Group Mean Normative Data .............................................................. 76

Plyometric Group Mean Normative Data ......................................................... 77

Plyometric Group Mean Normative..................................................................... 77

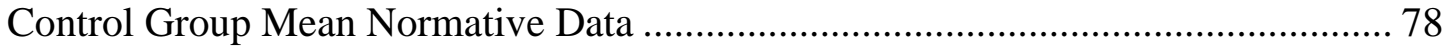

Control Group Mean Normative Data ........................................................... 78

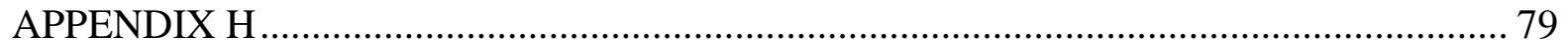

Progression Graph for Leg Press and Squat Jump............................................ 79 


\section{LIST OF TABLES}

Table $3.1 \quad$ Weekly Strength Training Program ......................................................... 29

Table $3.2 \quad$ Weekly Plyometric Training Program ...................................................... 30

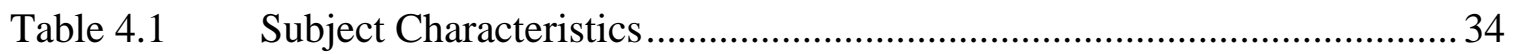

Table 4.2 Peak Knee Torque at Pre and Post-Testing During 8 Weeks of Resistance Training or Plyometric Training in Older Adults.................39

Table 4.3 Average Knee Power at Pre and Post-Testing During 8 Weeks of Resistance Training or Plyometric Training in Older Adults .................40 


\section{LIST OF FIGURES}

Figure 3.1 The AlterG Treadmill (photo courtesy of AlterG Inc.)............................ 32

Figure $3.2 \quad$ Specialized Shorts with Zipper for Air Seal ......................................... 32

Figure 4.1 Mean Times in Seconds for Sit-to-stand at Pre and Post-test.................. 35

Figure 4.2 Mean Times in Seconds for Stair Climb at Pre and Post-test.................. 36

Figure 4.3 Mean Power Output in Watts during Stair Climb at Pre and Post-test..... 36

Figure 4.4 Mean Estimated 1RM Relative to Body Mass. A. Leg Press, B. Leg

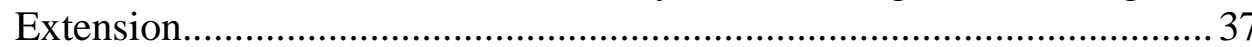




\section{CHAPTER 1: INTRODUCTION}

According to the National Institute on Aging, by 2050 the population of adults age 65 and older is expected to reach 1.5 billion people. ${ }^{1}$ This would account for $16 \%$ of the world's population compared to 2010 when it was only $8 \%$. The term baby boomer has increasingly been used to describe the prevalence of older adults living in today's population. Baby boomers are now considered a cohort of people that were born in the post-world war II period of 1946-1964. In the United States alone, by 2029, they will make up $20 \%$ of the population. ${ }^{2}$ As the number of older adults increases, investigations into improving overall health are warranted.

As humans age, muscular strength, "the ability of a muscle or muscle group to exert a maximal force," decreases and the loss of muscular power, "the optimal amount of work performed in a given time period," may be even greater. ${ }^{3,4}$ In older adults 65-89 years old, muscle strength has been shown to decline by up 1-2\% per year, whereas muscular power declines at an even higher rate of approximately $3-4 \%$ per year. ${ }^{3}$ Sarcopenia, the age-associated loss of skeletal muscle mass due to the reduction in muscle fibers and muscle fiber atrophy, has been previously found to play an important factor in age-related strength declines. ${ }^{5,6}$ This loss in muscle cross sectional area and subsequent muscle strength is dependent on a variety of factors: sedentary lifestyle and neurological, hormonal, nutritional, and immunological deficiencies. ${ }^{7}$ Largely, the $^{2}$ decrease in muscle mass and strength is due to older adults being less active. 
When exercise and activity decreases or ceases, muscle atrophies and strength declines which can lead to debility in independence and activities of daily living (ADLs) ${ }^{8,9}$ ADLs are described as everyday activities that people perform including selfcare such as feeding ourselves, bathing, dressing, etc. ${ }^{10}$ The ability to maintain independence and complete ADLs are of primary concern to older adults. ${ }^{11} \mathrm{With}$ the age-associated loss of strength and power, simple tasks such as reaching a dish on a high shelf, getting up from a chair, or ascending a flight of stairs can become daunting tasks.

Some ADLs involve tasks that may require balance as well as strength and power. Compromised strength, power, and balance can lead to increased fall incidence. ${ }^{12}$ Muscle power has shown a greater influence than strength on functional performance in older adults and that elderly fallers have less power in lower limbs in comparison to nonfallers. ${ }^{13}$ According to the Center for Disease Control (CDC) falls are the number one cause of morbidity and mortality in people age 65 and older, resulting in five times more hospitalizations than any other types of injuries. ${ }^{14}$ Falls account for nearly $90 \%$ of hip fractures in older adults, and of these, up to $20 \%$ become fatal. ${ }^{15}$ To put this into a financial perspective, in 2012 medical costs directly related to falls in older adults were estimated at 30 billion dollars. ${ }^{16}$ With the increase of older adults, by 2020 the estimated healthcare costs related to falls is projected at 67 billion dollars. ${ }^{16}$ Reducing fall risk is worthwhile undertaking for older adults, and with an associated decreased power in elderly fallers, a strength training program focused on power may be important for this population.

Several studies have shown that strength training for older adults can increase strength and reduce the number of falls through improving agility and dynamic 
balance. ${ }^{9,13,17-20}$ The American College of Sports Medicine (ACSM) current recommendations for strength training for older adults are vague and includes performing resistance training at least two days per week, but not more than four days, with 48 hours rest between training. ${ }^{4}$ They also recommend performing total body exercises that target large muscle groups with eight to ten exercises with one set of 10-15 repetitions. ${ }^{4}$ Strength training for older adults is an important part of their exercise prescription; however power training may have more practical outcomes.

Muscle power is related, but differs from muscular strength. Muscular power is the ability to perform work (force $\mathrm{x}$ displacement) per unit of time, meaning power is a product of strength and velocity (displacement/time). ${ }^{21}$ This means power encompasses both strength and velocity. Muscular power can be trained in many ways and varies from sport specific movements to normal daily living tasks. ${ }^{22}$ An example of a power training exercise for a college athlete is the variation of the Olympic power clean, in which an athlete lifts a barbell from the ground and explosively raises the bar up and catches it at the shoulder level in a squat position and then stands up. This common exercise is aimed at producing maximal force in the shortest amount of time. A power movement for an older adult for instance can be walking quickly, climbing a flight of stairs, or standing from a chair. ${ }^{11}$ Although a power clean and sit to stand movement are vastly different, they both require a relatively rapid force development for success.

A popular exercise technique focusing on increasing muscular power is plyometrics. Plyometrics are dynamic exercises that uses the stretch-shortening cycle to promote maximal power by performing an eccentric motion followed by a rapid concentric motion. ${ }^{23}$ An example of a popular plyometric is the squat jump, in which the 
subject performs a countermovement jump starting with a squatting motion followed by an explosive concentric motion, jumping as high as possible. ${ }^{24}$ Plyometrics have shown great benefits for increasing muscle force, power, and agility in adolescents and recreational adults. Agility is defined as the ability to change direction quickly with a minimal loss of control or speed. ${ }^{25}$ In a study of recreational athletes at least 18 years of age, it was found that a six-week plyometric training program significantly increased participants' agility when compared to a control group. Agility correlates well with balance thus having greater agility indicates improved balance. ${ }^{26}$ Plyometrics can also benefit jump height, an indicator of lower limb muscle power. ${ }^{27} \mathrm{~A}$ meta-analysis investigating the effect of plyometrics and jump height, found that plyometric training showed a significant and practically relevant improvement in vertical jump height. ${ }^{28}$ Older adults may not need the agility and jump height like that of a college athlete, but the associated increase in these two measures may lead to performing ADLs with more ease. $^{11,22}$

Although there is an increasing amount of evidence in support of plyometrics and its enhancement of muscular power, data is lacking on older adults. For example, a PubMed database search including the terms "older," "adults," and "plyometrics," yielded zero results. Plyometrics can be complicated and demanding movements that require great neuromuscular control, which may explain the lack of utilization in the older adult population. The most common forms of plyometric-type movements include box jumps, depth jumps, and squat jumps all of which involve some variation of bounding, hopping, and jumping. ${ }^{27}$ Level of fitness must be considered when prescribing plyometric exercise. The National Strength and Conditioning Association (NSCA) 
include in their guidelines that to properly perform lower body plyometrics proper technique, strength, speed, balance, and age is required. It is recommended that proper technique be demonstrated such as landing with a good center of gravity and that a participant be able to perform a one-repetition maximum (1RM) squat of at least 1.5 times their body weight. The participant should also be able to perform five repetitions of the squat at $60 \%$ body weight in five seconds or less. The NSCA also recommends balance standards be met - such as standing on one leg for 30s without falling. Due to these quite challenging guidelines and because of the difficulty for older adults to properly meet these guidelines, plyometrics are not typically prescribed for exercise.

The design of equipment to maximize functional movements in people with decreased strength, balance, coordination, and exercise capacity has become increasingly popular. The AlterG treadmill allows for older adults with decreases in balance and strength, or people recovering from injuries, to challenge their balance and functional capacity. The AlterG treadmill is an anti-gravity treadmill that lets the participants unweigh their body weight by up to $90 \% .^{29}$ This is accomplished through lower body positive pressure which uses positive air pressure applied within a sealed chamber surrounding the subject's pelvis and legs to support the user's body weight. ${ }^{30}$ These treadmills allow for improved mobility, strength, and safety while improving functional capacity related to endurance, strength, and power. ${ }^{31}$ Since its inception, the treadmill has been used in a variety of ways. One area that is of great interest is the use of the AlterG by older adults. Because of the stability and support participants experience while on the treadmill, falls can be prevented and participants can perform more complex and dynamic exercises with the benefit of unweighing at a certain percentage of body weight. 
A novel application of the AlterG treadmill that has little research support is for its use during plyometric exercises. These exercises can include single leg bounds, double leg bounds, and squat jumps. Relating back to the NSCA recommendations for the incorporation of plyometric exercise, this can dramatically reduce the necessary strength, speed, and balance required to perform such movements. Because of the stability and support that the treadmill provides, this may be an ideal situation for older adults to begin plyometrics and encounter the benefits of power and agility.

There is a need for more research utilizing a variety of protocols and options for exercise training by older adults. Specifically, ways to increase power and ADL abilities in this population are critical. Currently, there is a lack of research in the field of plyometric exercise and older adults. There is even less research using anti-gravity treadmills. The lack of research of plyometrics by older adults raises the question, "Can performing specific types of power movements in a safe, alternative method such as an AlterG treadmill allow for the benefits seen in prior research of adolescents and young adults?"

\section{Purpose}

Because of this lack in research of older adults and plyometrics the purpose of the study is to determine if performing plyometrics in an AlterG treadmill can lead to an increase in power output and functional movement in older adults as compared to traditional strength training.

\section{Hypothesis}

Since previous research has shown many benefits of plyometric exercise and power output, it is hypothesized that the capability of older adults to perform plyometrics 
in a safe, stable, and supportive machine such as the AlterG will increase power output in older adults when compared to control and resistance training groups as measured by three different tests including a repeated chair sit-to-stand, timed stair climb, and isokinetic extension/flexion.

\section{Significance}

The significance of this study is that it may give insight to practitioners that older adults performing power movements in a safe, stable, and supportive environment may increase their power output. Because of the tremendous benefits plyometrics have shown in adolescents and young adults, it is important to see if the same results are seen in older adults who utilize these movements. If an increase in power output can lead to greater balance and functional ability this may lead to several benefits such as reducing fall risk, increased independence, and performing ADLs as adults age. Training power movements in an AlterG treadmill may be a safer and more comfortable modality. 


\section{CHAPTER 2: LITERATURE REVIEW}

It is well known that as humans age, their strength, power, and muscle mass diminish. This leads to a multitude of negative consequences including reduced quality of life, decreased energy expenditure (both at rest and during exercise), and increased body fat which can lead to several other conditions such as metabolic disease. ${ }^{32}$ However, resistance training in older adults has been shown to increase strength and power, reduce the difficulty of performing ADLs, as well as improving energy expenditure and body composition. ${ }^{32}$ While traditional resistance training has been common in the exercise prescription for resistance training in older adults, there has been an increased focus on power training as a type of exercise because many functional tasks are more dependent on power than strength. ${ }^{22}$ Plyometric exercises have been shown to increase strength and power output, but have not been studied as often in older adults compared to younger, recreationally active adults. This review of literature will focus on bridging the gaps from strength training to power training to plyometric training, focusing on the benefits of each and in the older adult population.

Muscular power is defined as work (force $\times$ distance) divided by time, whereas strength is the ability to produce force. Thus, power incorporates both muscular strength and velocity of contraction to produce high amounts of force rapidly. ${ }^{22}$ Strength relies solely on the force generated without regard to the duration of the contraction. Power is comprised of both force and the rate of force development (RFD). RFD is determined by several elements including both central and peripheral factors including neural drive and 
muscle fiber type and size. ${ }^{33}$ RFD is important because most basic ADLs (such as rising from a chair or climbing a flight of stairs) do not rely solely on muscular strength, but also the RFD (i.e. muscular power). ${ }^{11}$ Power output in older adults differs greatly from that of college-aged adults. It has been found that power and force generation decline markedly with age as evidenced by decreased performance during chair rising and jumping tests. ${ }^{34}$ There have been suggested reasons for the power decrement that occurs with aging including an advanced atrophy of type II muscle fibers (which contribute more to power output than type I fibers) and the loss of muscle mass known as sarcopenia. $^{7,35}$

Several strength-training programs have been shown to increase power output in older adults..$^{3,11,13,17,18,36-38}$ Strength training studies range anywhere from 9 weeks ${ }^{39}$ to 84 weeks $^{40}$ and can greatly vary in design such as consisting of exercises involving only 2 lower limb exercises ${ }^{41}$ up to 10 total body exercises. ${ }^{20}$ It is well documented that traditional strength training can lead to increases in muscular strength and power, but focus should be shifted to explore ways to increase muscle power to a greater extent and various exercise protocols to do so. ${ }^{42}$

Plyometrics are defined by the National Strength and Conditioning Association (NSCA) as, "The stretch-shortening cycle (SSC), characterized by a rapid deceleration of mass followed almost immediately by rapid acceleration of the mass in the opposite direction." 23 The SSC has been explained as the agonist muscles being placed in a lengthened (eccentric) active state, prior to a rapid shortening (concentric) motion. The subsequent concentric motion of the muscle is enhanced due to the stored elastic energy and increased agonist muscle neural stimulation during the stretch. ${ }^{43}$ This movement has 
been likened to a rubber band as the person uses stored energy generated by the eccentric motion (stretching the rubber band), followed by a rapid concentric motion (releasing the rubber band) that generates great power. ${ }^{44}$ The quicker the muscle is stretched; the greater the amount of force that can be produced, therefore creating a more powerful muscle movement.

\section{Muscular Strength Training in Older Adults}

Muscular strength is believed to peak around the ages of 20 to 30, remain stagnant for a few decades and then begin to decline by the 50s with a more rapid decrease by age $65 .^{45}$ Due to this rapid decrease in muscular strength in older adults, comorbidities such as falls, a reduction in walking speed, an increased risk of disability, difficulties with activities of daily living (ADL), and physical inactivity may occur. ${ }^{46}$ It is estimated that one in three adults will experience a fall at least once per year after age 65. ${ }^{47}$ Being able to perform ADLs specifically related to balance, will not only maintain independence, but also reduce the risk of fall and injury; thus, cutting the cost of medical care and the subsequent loss in strength due to recovery-induced inactivity. ${ }^{12}$ Because of the growing number of adult fallers, it is important to understand the problems that arise with a decrease in muscle power as well as the functional abilities that suffer.

The decrease in muscle mass and strength is a well-known part of the aging process. ${ }^{11,39,41,45}$ Age-related sarcopenia (loss in skeletal muscle mass) has been hypothesized as one of the major reasons for decreases in strength and power ${ }^{5,8}$ While the age-related decrease in skeletal muscle mass has been considered a cause of muscle weakness, other factors such as changes in neural, hormonal, and habitual activity levels can also play a role. ${ }^{7}$ 
It has been shown that a progressive strength-training intervention can increase muscle strength that has been lost due to a decrease in physical activity. ${ }^{40}$ Progressive strength training is widely considered to be the preferred method for strength training, including in older adults. This involves working against an external force that is increased as muscular strength increases, known as the overload principle. ${ }^{46}$ Once an individual's maximum strength is determined either through traditional one rep max testing or though estimations, intensity can be set and exercises are performed through determined sets and repetitions several times a week, specific to the individual's goals. The current ACSM recommendations for older adults participating in strength training include one or more sets of 10-15 repetitions of moderate intensity (60\%-70\% 1RM). ${ }^{4}$ These strength-training programs have not only resulted in increased muscle strength but also improved functional abilities. ${ }^{11,17,20}$ As early as the 1980s, strength training focusing on older adults became of particular interest. In one of the earliest findings of substantial increases in muscular strength, size, and fiber composition, Frontera et $\mathrm{a}^{41}$ found that performing knee flexion and extension exercises at $80 \% 1 \mathrm{RM}$ three times per week for 12 weeks elicited strength gains of up to $226 \%$ in previously sedentary older adults. ${ }^{41}$ Additional studies investigated various strength training programs on older adults, many of them lasting as little as 8 weeks. ${ }^{48-50}$

Strength training studies in older adults have not been limited to short-term studies. In a longitudinal study published in 1996, 113 healthy older adults ranging in age from $60-80$ years performed a resistance-training program for two years. In this program, participants trained twice a week (with 24-48 hours of recovery between sessions) on military press, leg press, and bench press. They completed 2 sets of 10-12 
repetitions of each exercise at $50 \%$ of their $1 \mathrm{RM}$ initially, progressing to $80 \% 1 \mathrm{RM}$ "after a few weeks" of training. 1RM was reassessed at 6-week intervals and the training weight adjusted. They found that strength gains were still occurring into the second year of training, and at the same rate of the first year of training. They demonstrated an increase of $63 \%$ total change of 1RM strength in year one and $61 \%$ increase in year two. These studies demonstrated that progressive strength training studies with vastly different training lengths can all exhibit positive outcomes in older adults.

The strength training protocols used in previous studies have varied and strength nearly always improves. There have been studies that have focused on the lower body by performing knee flexion and extension, ${ }^{41}$ the leg press, ${ }^{40}$ or home-based body weight exercises. ${ }^{51}$ There have also been studies that focus on strength of the upper body such as the chest press, seated row, bicep curls, and circuit based training. ${ }^{20,37,39}$ In all of these studies muscular strength was increased, regardless of training protocol or exercises performed. Lower body strength has been seen to increase by as much as $32 \%$ in the leg press and high as $107 \%$ and $227 \%$ in the knee flexors and extensors, respectively.

The benefits of strength training go beyond just being able to lift more weight and exert more force. Several strength training studies have found that not only can muscular strength and size increase; improvements are also seen during balance and functional tasks. ${ }^{3,9,11,13,15,18,37,38,52}$ Some of the most common functional performance tasks in older adults include the timed sit-to-stand from a chair, ${ }^{18}$ ascending or descending stairs, ${ }^{3} 6$ meter timed walk, ${ }^{11}$ and balance assessment tests, such as the Berg Balance Scale. ${ }^{19}$ In a study investigating a battery of functional measures, older adults were found to improve their chair sit-to-stand by $28 \%$ and their stair climb time by $12 \% .{ }^{53}$ Other functional 
measures such as walking speed has also been shown to be increased up to $17 \%$ after a strength training intervention. ${ }^{49}$ It is clear that strength training in older adults can result in numerous benefits, including increased muscle mass, increased muscle size, greater muscle fiber composition changes, and greater functional abilities whether a short-term or long-term, upper body, lower body, or total body exercises are implemented.

\section{Muscular Power Training in Older Adults}

Research has shown that traditional resistance training can produce increased muscular strength as well as muscle size ${ }^{17,37,40,41,45,48}$ However, muscular strength and hypertrophy may not be the best indicators of function. For older adults, an increase in power could mean a decreased risk of falling, ease rising from a toilet, or climbing a flight of stairs. Tasks like these are more closely related to the mechanisms associated with muscular power. Muscular power (the product of force and velocity of muscle contraction) have been shown to be more indicative of functional health for older adults. ${ }^{11,54}$ As early as the 1990 s, peak muscle power has been studied as an outcome variable distinct from muscular strength. ${ }^{55}$ The increased research focus on the effects of power training for older adults has increased because of several studies showing a stronger relationship between functional movements and power. ${ }^{22}$ In one of the first power-specific training studies conducted, healthy, older participants were randomly selected to a power-training group or a walking group. The power training program was designed to train high velocity movements of the hip and knee extensor muscles. Three types of repetitions speeds were used: usual, ramped, or high velocity. Usual-pace consisted of the subjects performing a comfortable, but not slow pace. Ramped sets began with reps 1-3 at usual pace, reps 4-6 a little faster, and reps 7-10 at high-velocity or 
as fast as possible. After 3 weeks, ramped sets were used for the first set and highvelocity thereafter. While both groups improved leg strength (22\% power group, $12 \%$ walking group), the power training group made dramatic power gains of up to $150 \%$ improvements in leg press power at $70 \%$ of body mass, while walkers did not improve power. ${ }^{18}$ The findings of increasing power output from performing power specific, highvelocity strength training has led to several studies incorporating power-type movements into older adult exercise programs. These studies have found an increase in balance, functional performance, and muscular power such as improved balance measured by single-leg standing and sway, improved stair climb time, walking time, and chair standing. ${ }^{13,19,37,49}$ This type of training leads to decreased falls and ultimately a decrease in hospitalizations and mortality as well as an increase in independence for older adults.

With the demonstrated improvements in power through the use of high-velocity training programs in older adults, investigators began to examine the effects of power training programs on previously sedentary older participants. High velocity movements have been shown to increase muscular power when compared to slow velocity resistance training. In 2006, Bottaro demonstrated that inactive older males exercising twice weekly for 10 weeks could significantly improve measures of functional ability and muscular power. ${ }^{17}$ Participants were divided into two groups: the power training group performed 8-10 repetitions at $60 \% 1 \mathrm{RM}$ as fast as they could in the concentric action $\sim 1 \mathrm{~s}$, and $\sim 2-3 \mathrm{~s}$ in the eccentric action and the traditional training group performed exercises taking $2-3 \mathrm{~s}$ in both directions. The power training group improved measures such as the 30 -s chair stand test (43\% vs. $6 \%$ ) and the 8 -ft up-and-go test (15\% vs. $1 \%$ ) as well as muscular power calculated from the resistance machines as the force and speed of contraction in 
the chest press (37\% vs. 13\%) and leg press (31\% vs. $8 \%$ ) compared to the traditional training. ${ }^{17}$ This study supported evidence that older male adults can significantly improve muscular power and functional activities utilizing power training rather than traditional, low-velocity strength training while having similar increases in strength. Similarly, a study comparing traditional strength training to power-specific training showed a significant increase in the physical function of community dwelling, older adults. A strength-training group performed exercises at $80 \%$ of the $1 \mathrm{RM}$ while power training groups performed at $40 \%$ of their 1RM and performed the exercise movement as quickly as they could. The power training group performed less absolute work per exercise session yet was able to increase their physical functional status determined by the Continuous Scale Physical Function Performance Test (CS-PFP). This validated test measures a battery of 16 everyday, functional tasks. The power training group significantly improved their overall CS-PFP scores, balance and coordination measures, endurance, and flexibility compared to the strength training group. ${ }^{56}$ High-velocity training has demonstrated positive impacts on both functional tasks as well as muscular strength. Of particular interest is that the power training groups performing less absolute workload (total amount of weight lifted) but were still able to attain similar, if not better, strength gains compared to groups that are doing more work. (REF)

Direct comparisons of strength versus muscular power-specific resistance training have been established in older adults. This research has focused on many aspects of muscle performance in older adults, with the most commonly reported outcomes being muscle strength, muscle power, and batteries of functional performance tasks. Henwood and colleagues have demonstrated these findings in several studies. In 2005, it was 
shown that a group performing explosive concentric exercises twice a week for 8-weeks significantly improved their muscle strength anywhere from $21.4 \%$ in the leg press and up to $82 \%$ in leg curls, with an increase in upper body strength of $29.4 \%$ and lower body improvement of $42.6 \% .{ }^{57}$ The exercise group also significantly improved functional abilities of floor rise to standing, walking speed, and chair sit-to-stand..$^{37,57,58}$

In 2005, Henwood and Taaffe ${ }^{57}$ investigated power training versus only a control group. The researchers later sought to determine the most effective type of resistance training: high-velocity, slow-to-moderate velocity, or combined functional and highvelocity ${ }^{58}$ Older adults were assigned to either a high-velocity (HV) group that trained twice a week, slow to moderate-velocity (CT), once weekly of high-velocity and functional training $(\mathrm{CB})$ or no training $(\mathrm{CO})$. All three training groups significantly improved their muscular strength compared to $\mathrm{CO} .{ }^{58}$ However, during functional task measures, the HV group was the only group to significantly improve their sit-to-stand compared to $\mathrm{CO} .^{58}$ This study once again demonstrated similar improvements between groups, but a group performing power movements had better outcomes. ${ }^{58}$

Finally, Henwood directly compared strength versus power resistance training in a large sample of older adults. The participants exercised twice per week in either a highvelocity or constant (strength) training group. After 24 weeks, muscular strength improved similarly between the training groups, with $51 \%$ and $48.3 \%$ improvements in strength for high velocity and strength, respectively. ${ }^{37}$ Peak muscular power also improved in both groups with a 50.5\% and $33.8 \%$ increase in high-velocity and strength groups, respectively. ${ }^{37}$ Functional performance including chair sit-to-stand, stair climbing, and 6 meter fast walk, was also increased in both groups. ${ }^{37}$ Again, this study 
demonstrated that muscle strength, power, and functional abilities could be similarly improved with both power and strength training. The importance is that the improvements in the power group occurred with less total work during training sessions. $^{37}$

A major concern that occurs with the aging process for many people is the loss of independence due to a decrease in functional ability. Not only has an increase in power output been shown to be possible in healthy older adults ${ }^{18,36}$ and inactive older men, ${ }^{40}$ but studies have shown that even frail, long-term residential care residents can increase their strength and power. ${ }^{11,37}$ Thirty long-term care facility older adults 75-94 years of age performed muscle power movements during a 10-week, progressive training program involving only Therabands (resistance bands) and body weight exercises. The authors found that participants were able to increase not only their knee extensor strength and power measured via isokinetic dynamometer by up to $60 \%$; but more importantly, functional activities such as the 8-ft up-and-go, chair stand, and 6-m timed tests improved $31 \%, 66 \%$, and $33 \%$, respectively. ${ }^{11}$

Muscular power training has been shown to increase muscle strength, power, and functional abilities. Another variable that commonly affects older adults are balancerelated challenges. According to the Center for Disease Control, medical costs directly related to falls in 2013 were estimated at $\$ 34$ billion. ${ }^{16}$ With the enormous financial burden that are associated with a falls, additional research are needed to develop programs for improving balance in older adults. In one of the more robust balance and power training studies in older adults, Orr and colleagues showed that using power training resistance exercises could increase balance in older adults utilizing a low load, 
high velocity approach. ${ }^{13}$ Participants were randomized to either a high, medium, or low intensity training group exercising at $80 \% 1 \mathrm{RM}, 50 \%$, or $20 \%$, respectively. A control group continued their normal daily habits with no resistance training. After 10 weeks of training, all groups significantly improved their peak power, strength, and endurance compared to control. ${ }^{13}$ It was shown that low intensity could improve power by up to $14 \%$, strength $13 \%$ and endurance $82 \% .{ }^{13}$ The high intensity group had higher changes in strength and endurance of $20 \%$ and $185 \%$, respectively. ${ }^{13}$ Interestingly, the low intensity group had the greatest improvements in balance scores, measured by force plates determining body sway and single leg standing. This showed that using lower loads and producing more rapid movements of the exercise produced a greater outcome in balance tasks. $^{13}$

Research on increasing muscle power and functional ability has been limited to resistance training utilizing low loads in a rapid, high-velocity approach. A major barrier to performing power training in older adults is the risk for injury. Instructions for older adults participating in resistance training programs typically include moving the weight in a slow and controlled fashion, which is contrary to the protocols used during power training. To produce these power movements, exercises should be performed using light to moderate weight, and utilizing explosive action and high velocity. ${ }^{22}$ With this in mind, other forms of exercise that focuses on increasing muscular power, such as plyometrics, have been more common in training programs for young, healthy adults. ${ }^{26,27,59,60}$ The benefits of plyometric exercise include the ability to perform movements that target power without the need of resistance training equipment. In addition, many of these exercises can also incorporate more functional movements such as balance and agility. 
While plyometrics have been shown to be beneficial in populations such as adolescents and young, healthy adults, these types of programs are rarely, if ever, implemented into the exercise prescription for older adults. Although there is less evidence supporting muscular power training in older adults than muscular strength, emerging research is beginning to shift towards resistance training methods that focus more on power rather than strength. It has been hypothesized that this is because measurement of muscular power in a single, explosive movement is more difficult than any type of strength measure. ${ }^{18}$ As discussed previously, more researchers are finding muscular power to be beneficial for increasing both strength and power. It is becoming more evident that a decrease in muscular power is more predictive of disability. Thus, it is important to determine whether power training is superior or comparable to strength training. ${ }^{61}$

\section{Plyometrics}

Plyometrics are a popular form of sport performance training and physical conditioning in young, healthy individuals. ${ }^{62}$ Because this type training incorporates the SSC, it has the potential to enhance both neural and the musculotendinous systems to produce maximal force in the quickest amount of time. Due to these enhancements, plyometrics have been used extensively to improve athletic performance such as vertical jumping; with several studies, reviews, and meta-analysis' proving its efficacy. ${ }^{44,59,60,63}$ More recently, investigators have looked into the positive benefits plyometrics have on neuromuscular function, explosive and endurance events, improving biomechanical technique, and potential positive bone adaptations. ${ }^{62}$

Previous research on plyometric training has found benefits and improvements in performance in children, ${ }^{59}$ college-aged men, ${ }^{27}$ and college-aged women. ${ }^{64}$ These 
improvements include improved vertical jump height, improved agility, and increases in bone-mineral density. There are several types of plyometric exercises. The most common plyometric exercises including bounding and jumping-type movements, such as squat jumps, countermovement jumps, countermovement with arm swing, and drop jumps. All of these movements require an eccentric motion followed by a rapid, concentric motion performed in the shortest amount of time to produce muscular power and optimally complete the movement. These types of movements are considered intense, complex, and require great neuromuscular control, which may be reasons that they are not utilized more often by older adults. The majority of the research on plyometrics has been focused on young, healthy adults. In a meta-analytic review of the effects of plyometric training on performance, it was found that performing plyometric exercises can significantly increase vertical jump height with an effect size of 0.88 in the countermovement jump. ${ }^{28}$ The mean age of participants in this analysis was 23 years old, with a range of 13-29 years of age. Although positive, these findings may not be relevant to older adults. Further research on this topic is needed.

There have been several studies that have investigated the effects of plyometrics on strength and power of the lower body. In a review of the neuro-musculoskeletal and performance adaptations to plyometrics, it was found that maximal strength results are variable ranging from a $3.2 \%-45.1 \%$ increase in strength, with mostly positive effects of greater than $10 \% .{ }^{62}$ In the same review, it was found that in 13 of 16 studies included, increases in muscular power occurred by $2.4-31.3 \%{ }^{62}$ Most of the measures included sport specific tests such as vertical jumping or bounding. Strength and power are related because both are necessary for optimal performance. These are important findings 
because they demonstrated that regardless of the age, training status, or sex of the subjects, plyometric interventions produce increases in strength and power. ${ }^{60}$

It should be noted that in several studies, a combined intervention involving both plyometrics and resistance training can produce similar or greater results than plyometrics alone. In a study on 12-15 year old boys, one group performed plyometrics involving jumps, hops, and bounds as well as resistance training that involved total body exercises while a second group performed only the resistance training exercises. After training twice a week for 6 weeks, it was found that the group that performed the combination of plyometrics and resistance training significantly improved their vertical jump by $8.1 \%$, long jump $6 \%$, shuttle run $3.8 \%$, medicine ball toss $14.4 \%$, and flexibility $27.6 \%$, while the resistance training group significantly improved during ball toss by $5.6 \%$ and flexibility tests $29 \%$ only. ${ }^{59}$ The combination group was significantly higher than resistance training alone in the long jump, shuttle run, and ball toss. While this study was in younger boys, it found similar results as a study of adults that found vertical jump increases of $15 \%$ when combining resistance training and plyometrics compared to $11 \%$ and $9 \%$ when performing only resistance training or plyometrics, respectively. ${ }^{65}$ It has been suggested that the combination of resistance training and plyometrics may be synergistic, that is the combination of each may have greater outcomes than performing each alone. Authors have also posited that plyometrics can act as a primer of the neuromuscular system, recruiting more neural pathways during exercise. ${ }^{59}$

An interesting area of research of implementing plyometrics training programs is effects of bone adaptation. It has been well established that physical exercise has a positive effect on bone mass. ${ }^{62}$ Because plyometrics are high impact exercises which can 
be associated with high ground reaction forces reaching upwards of 7 times bodyweight, this type of exercise may be suitable for increasing bone mass. ${ }^{62}$ Bone density decreases with age and osteoporosis is a major concern for older adults. There have been several studies investigating the effect of plyometrics on bone adaptation. Unfortunately, these have been mostly examined on children, but have found relative gains of $1-8 \%$ in bone mineral density, bone mineral content, or bone area. However, these effects are not homogenous and depend on the skeletal site and age. These increases are also greater at different bone sites such as higher contents in the femoral neck compared to the lumbar spine. ${ }^{62}$ There have been studies in adults, but to a much lesser extent, finding positive changes in bone mass has been observed in young and pre-menopausal women, but not in post-menopausal women. ${ }^{66}$ More research investigating the effect of plyometrics on bone adaptation in older adults is suggested.

The question arises as to the applicability are these types of exercises for older populations. If plyometric movements can increase strength, power, balance and agility, and bone mass in young adults, then older adults may be able to achieve similar benefits through plyometric training programs. However, because power training in older adults has typically utilizes some form of resistance training and the velocity of movement as the type of exercise, plyometrics have not been utilized. This may also be attributed to the NSCA guidelines on performing these exercises, in which researchers may not feel comfortable with having older adults perform.

The literature on plyometrics is generally positive as noted by the previously mentioned meta-analysis on the effects of plyometric training. With advancing technology that allows for people with both injuries and disabilities to exercise using 
pneumatic, unweighting treadmills and AlterG treadmills, the possibility for older adults to participate in plyometric exercises has increased. Using these new types of modalities may shed light on the possibility of older adults obtaining the same benefits of power output as their younger cohorts.

\section{Anti-gravity Treadmills}

The AlterG anti-gravity treadmill is a treadmill that utilizes differential air pressure to alter and ultimately reduce the weight of an individual up to $80 \%$ of original body weight while on the treadmill. ${ }^{29}$ These treadmills use lower body positive pressure, which forces positive air pressure applied within a sealed chamber surrounding the subject's pelvis and legs to support the user's body weight. ${ }^{29}$ A study by Patil showed that unweighing in an anti-gravity treadmill was effective in reducing the amount of ground reaction forces generated while running. ${ }^{31}$ Due to this unweighting of a percentage of bodyweight, the level of impact is reduced and may allow for people with injuries such as pelvic fractures or Achilles tendon surgery to begin rehabilitation sooner. ${ }^{67,68}$ The metabolic demand of anti-gravity treadmills has also been shown to not significantly differ from traditional treadmills and that cardiovascular training can be achieved while exercising at a reduced body weight. ${ }^{29}$ While the research may be limited on this relatively novel training equipment, the ability to both reduce the ground reaction forces and achieve cardiovascular training is promising. Older adults may benefit from plyometric training protocols using the AlterG treadmill to accomplish benefits of power improvements previously seen in younger, recreationally adults. Participants are also attached to the equipment, making the risks of tripping or falling very low. If one can exercise at a lower percentage of their body weight, while attached in a safe and stable 
environment, the guidelines for performing plyometrics become much more feasible for this population.

\section{Summary}

This review of the literature examined the research of muscular strength training in older adults. There is overwhelming support for resistance training to promote muscular strength for older adults. The increased interest in muscular power as an indicator of older adults' functionality was also reviewed. While muscular power may now be considered a more important factor in healthy aging, research is lacking as to how to promote the greatest power output gains in this population. Manipulating strength training protocols by increasing the velocity at which they perform the exercises has been the most reliable method of generating increased power in older adults to date. While plyometrics, which involve the SSC, have been shown to greatly improve power output in adolescents and adults, the literature is lacking in this area in older adults. In a database search in the Journal of Strength and Conditioning Research and PubMed, the terms "older," "adult," and "plyometric," retrieved only articles studying youth, collegeaged, and athletes. Because of this gap, it is crucial to explore the plyometric option that may benefit the growing older adult cohort. The advancement of technology such as AlterG Treadmills, which allow the unweighing of a portion of body weight may allow for a safer modality of performing these types of exercises for older adults. 


\section{CHAPTER 3: METHODS}

\section{Participants}

Twenty-three (20 female, 3 male) older adults between the ages of 51-80 were recruited for this study. Participants were recruited from the Osher Lifelong Learning Institute, St. Alphonsus Hospital volunteer office, and word of mouth. Flyers were placed in the Osher Lifelong Learning Institute located at Boise State University as well as in their electronic newsletter and in the St. Alphonsus hospital volunteer office (Appendix A and B). Enrolled participants were also used as recruitment via word of mouth. Exclusion criteria included any resistance training for the previous 6 months, uncontrolled diabetes or uncontrolled hypertension, any known cardiac event, any history of orthopedic joint replacement surgery, use of any type of mobility aid (walker, cane, etc.) or any physical impairment that limits their mobility. Prior to participating in the study, each subject completed a health history questionnaire (Appendix C), and informed consent form (Appendix D and E) approved by Boise State University's Institutional Review Board. Participants were also required to obtain a signed physician's consent to participate before enrolling (Appendix F). All testing and exercise sessions was completed at the Human Performance Laboratory in the Norco Health Sciences Building and the Kinesiology Annex Gym on the Boise State University Campus.

\section{Procedures}

The study consisted of 27 visits over the course of 8 weeks, with each visit ranging from 45-90 minutes. 


\section{$\underline{\text { Visit 1: Orientation (45-60 min) }}$}

Prior to the initial visit, signed physician consent to participant was received. The initial visit involved meeting with the potential participants at the Boise State University Human Performance Laboratory and consisted of completing paperwork and orientation to the lab and equipment to become familiar with the testing. First, a health history questionnaire using guidelines set forth by the ACSM was completed to determine appropriate inclusion/exclusion criteria. ${ }^{4}$ Once eligibility had been verified, all study procedures were explained to the participant and they were given an opportunity to ask any questions before signing the informed consent. Then, participant height to the nearest $0.1 \mathrm{~cm}$ (calibrated stadiometer, Seca, Chino, CA.) and weight to the nearest $0.1 \mathrm{~kg}$ (digital scale, Tanita Corporation, Arlington Heights, Illinois) were measured. Next, the participants completed the equipment and training orientation. Demonstrations on proper form and technique were provided by the research staff. Participants were asked to perform submaximal efforts on the exercises in order to become familiar with the equipment, exercises, and facility. Participants were shown a demonstration of and practiced the three outcome measures: the sit-to-stand, stair climb, Biodex isokinetic dynamometer and strength tests which included the seated leg press, seated leg extension, and single leg lunge. Participants were encouraged to ask questions about any of the tests, lifts, or general inquires.

\section{Visit 2: Pre-Tests (60-90 minutes)}

Visit two consisted of baseline (pre-testing). After completing a second orientation and familiarization to the tests and equipment, all dependent measures were taken. Participants performed a 5 to 10 minute warm-up consisting of walking on a 
treadmill, followed by submaximal trials of each test. The order of testing was: body composition, sit-to-stand, stair climb, isokinetic measures, and strength tests.

Each participant performed a 5-repetition sit-to-stand protocol as fast as possible. Procedures established previously were utilized. ${ }^{69}$ With their arms folded across their chest, participants were asked to stand up from $43 \mathrm{~cm}$ tall chair until the legs were fully extended and sit back into the chair with buttocks touching the chair as fast as possible for a total of 5 repetitions. ${ }^{70}$ The timing began when the researcher announced, "Go", and ends when the participant's buttocks touched the chair for the $5^{\text {th }}$ time. A demonstration as well as a practice trial was permitted. Time was measured with a handheld stopwatch to the nearest 0.1 second. The best time taken from two trials was recorded.

The second test was the timed stair climb. The stair climb is an inexpensive and quick test that has been shown to be an indicator of leg power. ${ }^{71}$ After a five minute rest, participants were asked to ascend a flight of stairs consisting of nine steps each measuring $17 \mathrm{~cm}$ tall as quickly and safely as possible. Power output was calculated by multiplying acceleration due to gravity by participant body weight by vertical height in meters divided by time ([9.81 x BW x 1.53m]/time). Participants were allowed to rest for up to five minutes and then repeat the test. Timing was measured to the nearest 0.1 second with a handheld stopwatch. The better of the two times was recorded.

The next test performed was the isokinetic knee extension/flexion test. The participants followed previously established protocols for the Biodex isokinetic dynamometer (Biodex Medical Systems Model B-2000, Shirley, NJ). ${ }^{72,73}$ Each participant was seated on the Biodex chair secured with chest and thigh straps with the hip angle at 85 degrees. A pad was strapped $2-3 \mathrm{~cm}$ above and proximal to the malleoli 
across the shin. Participants then performed five repetitions each at three different velocities: $60^{\circ} / \mathrm{sec}, 120^{\circ} / \mathrm{sec}$, and $180^{\circ} / \mathrm{sec}$. Before each trial, participants completed three submaximal repetitions at the three different speeds to become accustomed to testing procedures. Participants were instructed to give maximal effort and strong vocal encouragement was given throughout the trials. The peak torque and average power were measured at all three velocities for both knee flexion and knee extension. Participants were allowed five minutes to rest in between each velocity testing.

Strength measurements during three different exercises were also assessed for each participant. (For this test, an estimated 1 repetition maximum (1RM) was measured by performing a 3-5RM in the plate loaded leg press, single-leg lunge using a Smith machine, and machine leg extension). Participants performed each exercise using a light weight for 10 repetitions to gauge intensity and evaluate range of motion. Once completed, participants rested for 3-5 minutes and a weight was estimated by the researcher to reach no more than five repetitions. If the participant performed five repetitions, increments of 5-20 pounds was be added until the participant could not reach 5 or more repetitions. The participant performed no more than three sets of the exercise to prevent fatigue from being the max factor. The $1 \mathrm{RM}$ was then estimated from the lifts and was used during the strength training program to assign appropriate weight percentages. ${ }^{4,74,75}$ The equation incorporates both weight lifted and repetitions met: [(100*weight lifted)/(102.78-(2.78*reps) $].{ }^{75}$

Group assignment was randomly assigned after all pre-testing sessions were completed to reduce any potential bias. The randomization scheme was generated via the web site randomization.com (http://www.randomization.com). 


\section{Visits 3-26 (30-45 minutes)}

Visits 3-26 consisted of the strength and plyometric training visits. The SG and PG participants came to their designated training location 3 times per week for 8 weeks to complete 24 training sessions. Training sessions were separated by at least 48 hours rest for three training sessions per week. Training logs were kept by the researcher with pertinent information for each exercise including amount of weight and reps performed. Before each training session, all participants performed a general warm up consisting of either walking on a treadmill or cycling on an ergometer for 10 minutes. Strength training sessions were performed by protocols set forth by ACSM. ${ }^{4}$ All exercises were performed at 3 sets of 10 reps at $65-80 \%$ of their 1RM. Rest periods were 60 seconds between sets and up to two minutes between exercises. In order to keep exercises between both groups as comparable as possible, only lower body exercises were utilized in the strength training group. Once a general warm up had been completed, SG completed 3 sets of 10 repetitions on the leg press, on the leg extension, and of single leg lunges (See Table 3.1). Progression for the SG followed the protocol that once the participant could complete all three sets and repetitions for the exercise, 5lbs were added.

\section{Table 3.1 Weekly Strength Training Program}

\begin{tabular}{lccc} 
& Monday & Wednesday & Friday \\
\hline Leg Press & $3 \times 10$ & $3 \times 10$ & $3 \times 10$ \\
Leg Extension & $3 \times 10$ & $3 \times 10$ & $3 \times 10$ \\
Single Leg Lunge & $3 \times 10$ & $3 \times 10$ & $3 \times 10$ \\
\hline Intensity was set at $65-80 \%$ & $1 \mathrm{RM}$
\end{tabular}


The PG followed the same protocol as SG but with different exercises. PG completed 3 sets of 10 repetitions of squat jumps, single leg bounding, and explosive skipping (See Table 3.2). These exercises were chosen because of the similarity between the strength training groups and to keep specificity of training as comparable as possible. These exercises are generally considered to be good beginner exercises when starting a plyometric training program. ${ }^{63}$ Progression for the PG followed the protocol of beginning at an agreed on percentage of unweighing of the individual so they could properly complete all sets and repetitions, and then increased the weight of the individual by $1 \%$ increments. CG did not perform any strength or plyometric training exercises during the study. The CG was instructed to continue their daily habits and to maintain their current activity status. Every effort was made to keep volume between the two training groups equal by utilizing similar exercises as well as keeping the sets and reps consistent. For example, the PG performed squat jumps and the SG performed a seated leg press, mimicking movements between the two exercises. Verbal instruction was also given to both groups. For the SG, they were instructed to give maximal effort and to keep proper form and complete maximal range of motion. For the PG, they were instructed to also give maximal effort in all their jumps. They were told to jump has high as they could ("try to jump out of the machine").

Table 3.2 Weekly Plyometric Training Program

\begin{tabular}{llll}
\hline & Monday & Wednesday & Friday \\
\hline Squat Jump & $3 \times 10$ & $3 \times 10$ & $3 \times 10$ \\
Single Leg & & & \\
Bound & $3 \times 10$ & $3 \times 10$ & $3 \times 10$ \\
Power Skips & $3 \times 10$ & $3 \times 10$ & $3 \times 10$ \\
\hline
\end{tabular}


Intensity was set at a $\%$ of body mass; Single leg bound performed 3x 10 on both legs; Power skips performed $3 \times 10$ on both legs at once, for a total of 20 repetitions each set. Visit 27: Post-Testing ( 90 minutes)

The last visit consisted of post-testing that were the same measures performed in visit two. Participants had body composition measured and performed the sit-to-stand, stair climb, isokinetic tests and strength measures, in that order. Procedures and rest time were identical to visit two.

\section{AlterG Treadmill}

The AlterG treadmill device (AlterG® Anti-Gravity Treadmill ${ }^{\circledR}$, AlterG® P200, Fremont, CA) was a novel piece of equipment to every participant. Therefore adequate time was spent explaining in detail how the AlterG works. Air is blown into a chamber installed over a standard treadmill (Figure 3.1), with patients using a specialized pair of shorts (Figure 3.2) that zip into the chamber, making an air-tight seal from the waist and below. Patients are therefore allowed to exercise on this treadmill without full force when air is blown in, which can be adjusted to reduce body weight (BW) as much as $80 \%$, that is, using only $20 \%$ of their BW. ${ }^{29}$ 


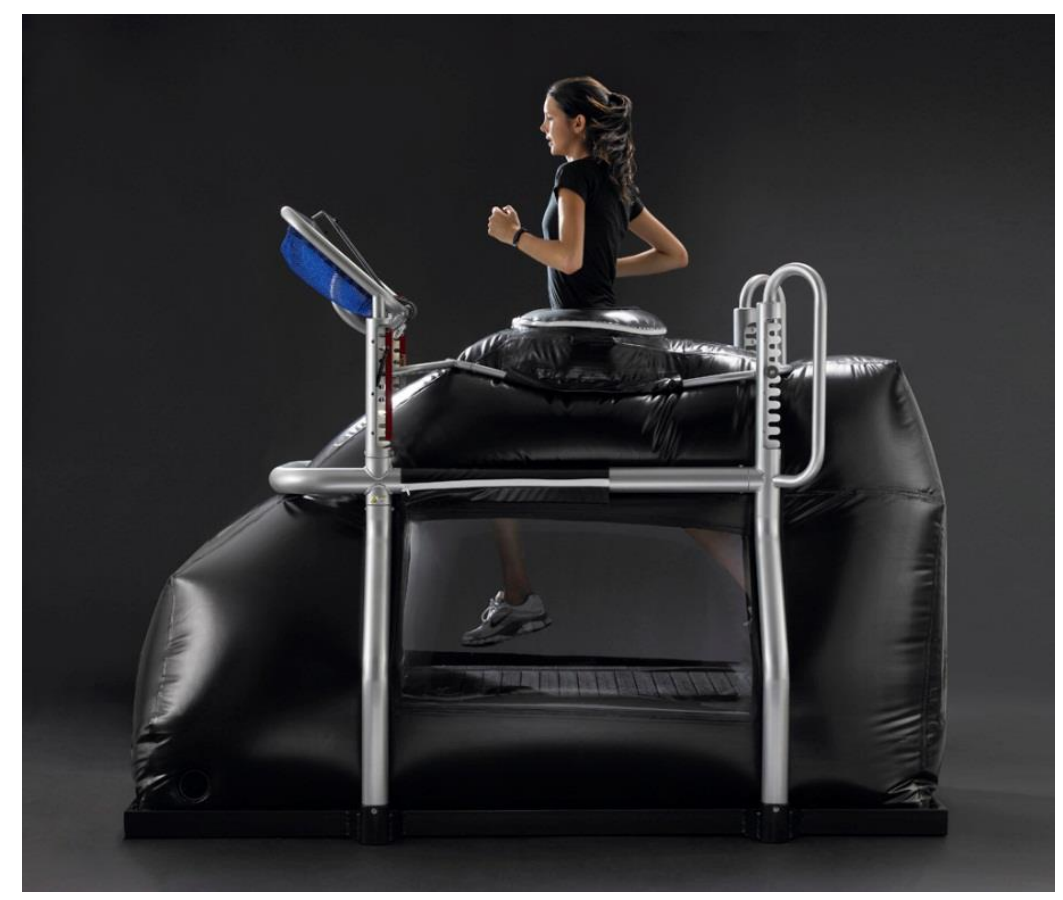

Figure 3.1 The AlterG Treadmill (photo courtesy of AlterG Inc.)

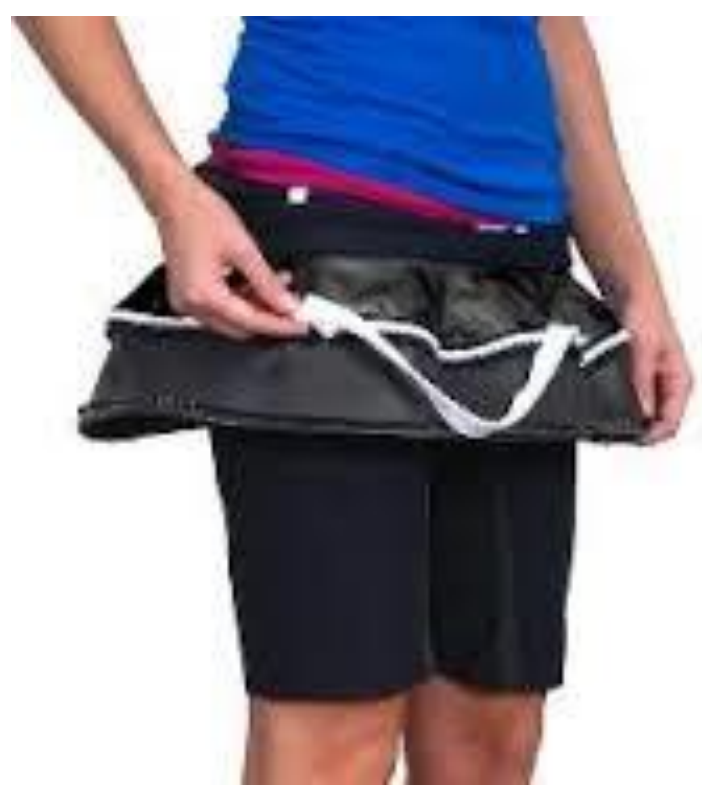

Figure 3.2 Specialized Shorts with Zipper for Air Seal (photo courtesy of AlterG Inc.)

\section{Statistical Analysis}

Descriptive statistics were reported for all variables. Analysis of variance (ANOVA) was used to determine if any differences existed between groups at pre- 
testing. A 2 x 3 (time $\mathrm{x}$ group) repeated measures ANOVA was used to determine if there were any changes over time between the groups. When appropriate, further post-hoc analysis was completed using a one-way ANOVA on the difference in scores with the Bonferroni adjustment was used to determine group differences between pre- and posttesting. Percent change in scores was calculated on the individual data as [(postpre)/pre] x 100, and the mean of the group change was reported. Significance level was set at 0.05. All analysis was completed on SPSS 23.0 (SPSS Inc., Armonk, NY). 


\section{CHAPTER 4: RESULTS}

The purpose of this study was to determine if performing plyometrics in a weight supported treadmill would lead to greater improvements in functional measures and power output compared to traditional strength training in older adults. Twenty-three participants completed the study. Within the study there were 3 groups: strength training group (SG), plyometric training group (PG), and a control group (CG) with 8,8 , and 7 participants in each group, respectively. Exercise session adherence was excellent with an average of $91 \%$ attendance (21/23 exercise sessions) with no participant missing more than 4 visits and no missed test days. Participant's physical characteristics are displayed in Table 4.1. There were no statistical differences in age, height, mass, body fat, or BMI between groups. Mean data in absolute values (Appendix G) was then made relative to participant body mass and was used for data analysis.

\section{Table 4.1 Subject Characteristics}

\begin{tabular}{lllll}
\hline & Strength & Plyometric & Control & $p$ value \\
\hline Age $(\mathrm{yr})$ & $66.25 \pm 7.09$ & $64 \pm 6.93$ & $62.71 \pm 9.46$ & 0.678 \\
Sex $(\mathrm{F} / \mathrm{M})$ & $6 / 2$ & $7 / 1$ & $7 / 0$ & \\
Height $(\mathrm{cm})$ & $165.44 \pm 6.60$ & $165.81 \pm 9.64$ & $166.50 \pm 5.44$ & 0.963 \\
Body Mass $(\mathrm{kg})$ & $80.12 \pm 16$ & $71.53 \pm 15.17$ & $67.96 \pm 12.28$ & 0.273 \\
Body Fat $(\%)$ & $37.94 \pm 6.90$ & $34.54 \pm 9.88$ & $37.43 \pm 4.69$ & 0.636 \\
BMI $\left(\mathrm{kg} / \mathrm{m}^{2}\right)$ & $29.27 \pm 6.02$ & $25.82 \pm 4.06$ & $24.53 \pm 4.53$ & 0.173 \\
\hline
\end{tabular}

Values are mean \pm SD 


\section{Functional Strength and Power}

For the chair sit-to-stand and stair climb (time and watts), repeated measures ANOVA found a significant group $\mathrm{x}$ time interaction effect $(\mathrm{p}<0.05)$. Post-hoc analysis for the chair sit-to-stand and stair climb (time) demonstrated that PG was significantly faster than CG $(p<0.05)$. There were no other differences between groups $(p>0.05)$ (See Figures 4.1 and 4.2). A significant effect for time was also found in the stair climb for power output in watts $(\mathrm{p}=0.001)$. Both the $\mathrm{SG}(\mathrm{p}=0.035)$ and $\mathrm{PG}(\mathrm{p}<0.001)$ had a significantly higher power output compared to CG, while SG and PG did not differ (See Figure 4.3).

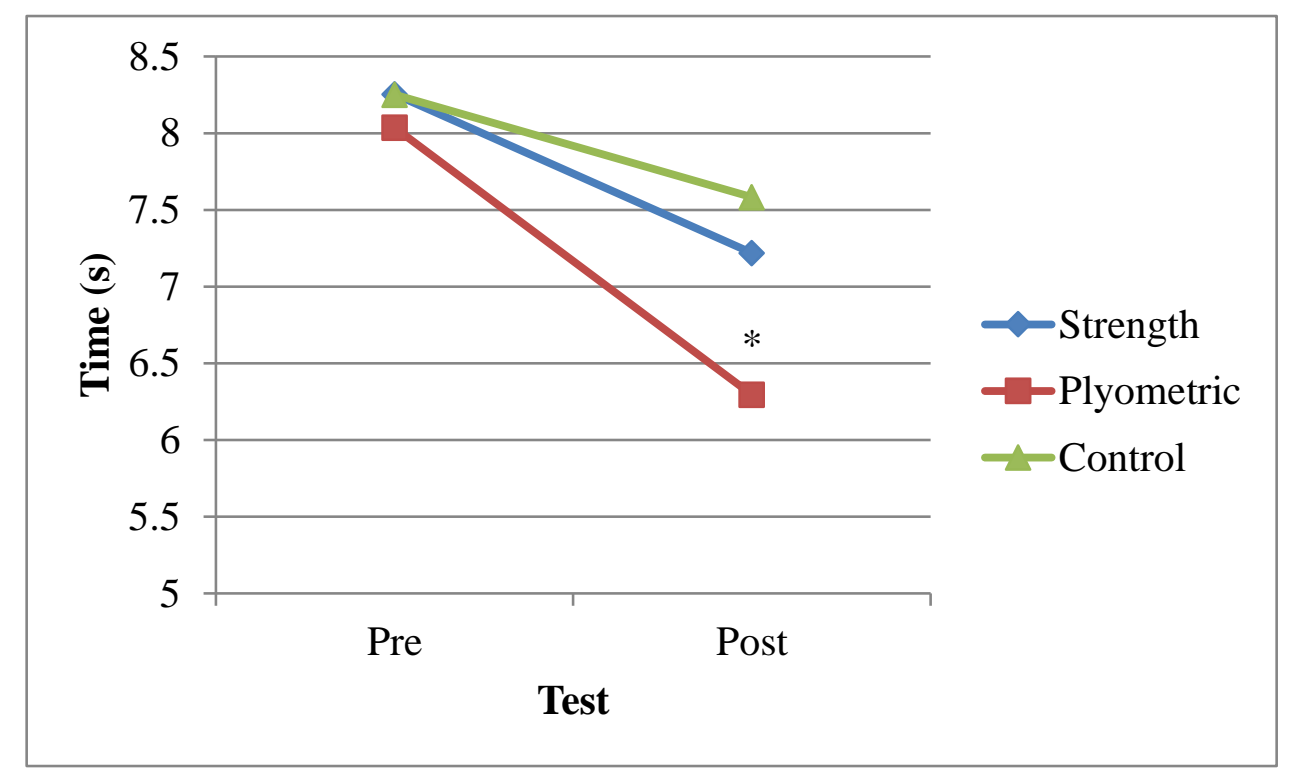

Figure 4.1 Mean Times in Seconds for Sit-to-stand at Pre and Post-test. *Significant difference between PG and CG $(p=0.013)$ 


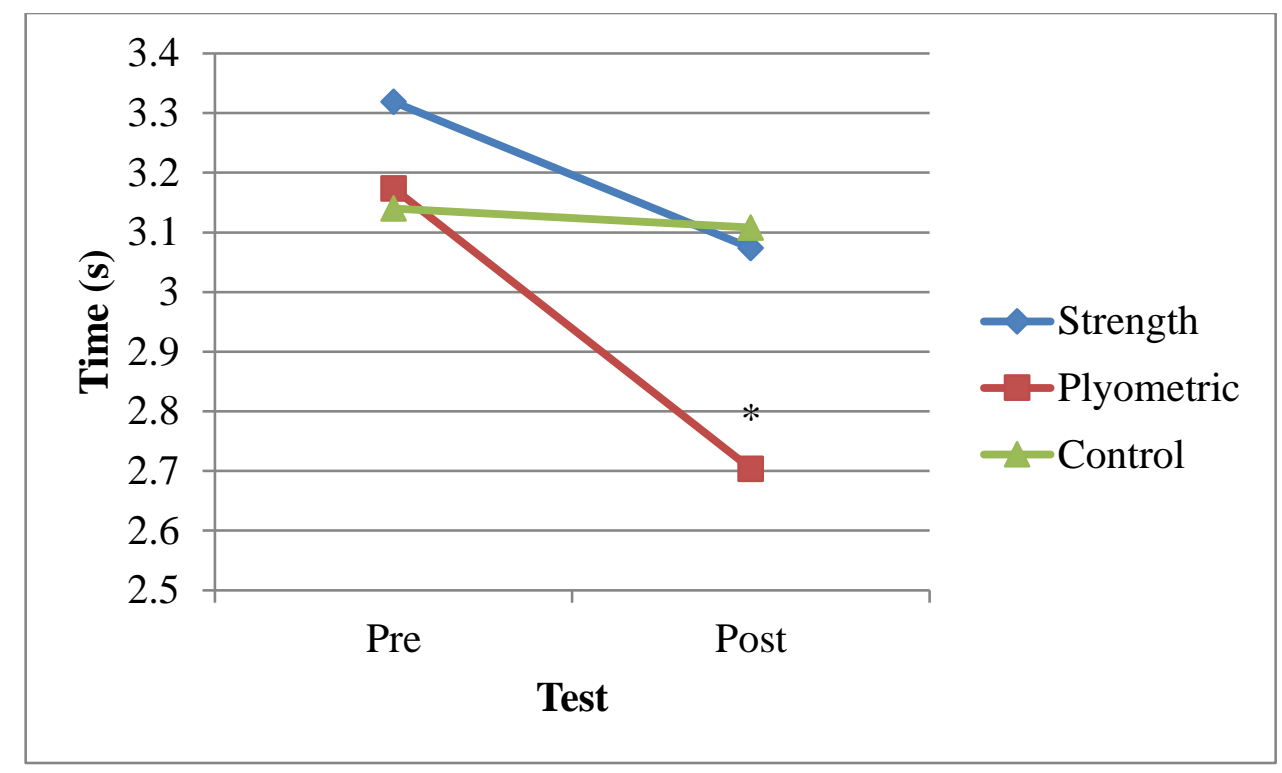

Figure 4.2 Mean Times in Seconds for Stair Climb at Pre and Post-test. *Significant difference between PG and CG $(p=0.002)$

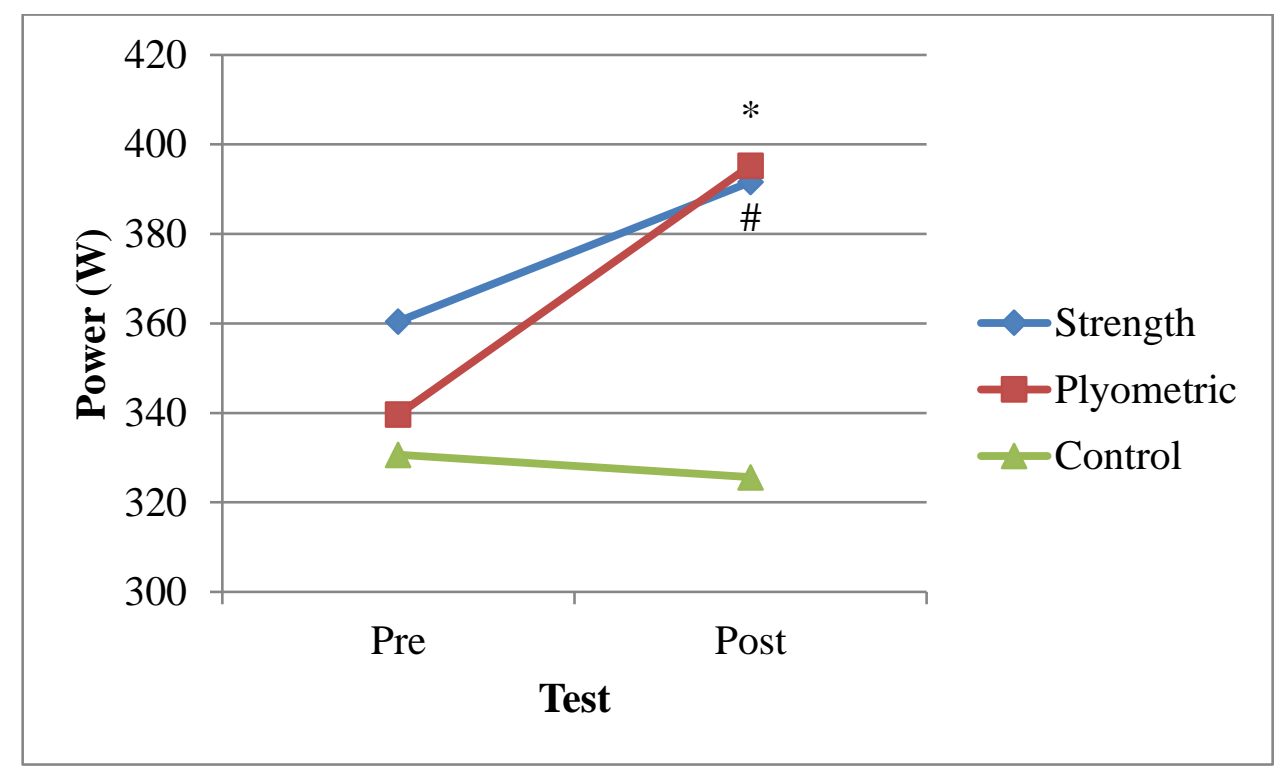

Figure 4.3 Mean Power Output in Watts during Stair Climb at Pre and Posttest. *Significant difference between PG and CG $(p<0.001)$. "Significant difference between SG and CG $(p=0.035)$

\section{Muscular Strength}

For the leg extension and single leg lunge, there was a significant group $\mathrm{x}$ time interaction $(p<0.01)$. In the leg extension PG was significantly higher $(p=0.009)$ than CG. SG was also significantly higher than CG $(\mathrm{p}=0.007)$. There was no difference 
between PG and SG in the leg extension ( $p>0.05)$. In the single leg lunge, SG was significantly higher than $P G(p=0.011)$ and $C G(p<0.001)$. PG was also significantly higher than CG $(\mathrm{p}=0.03)$ (See Figure 4.4).
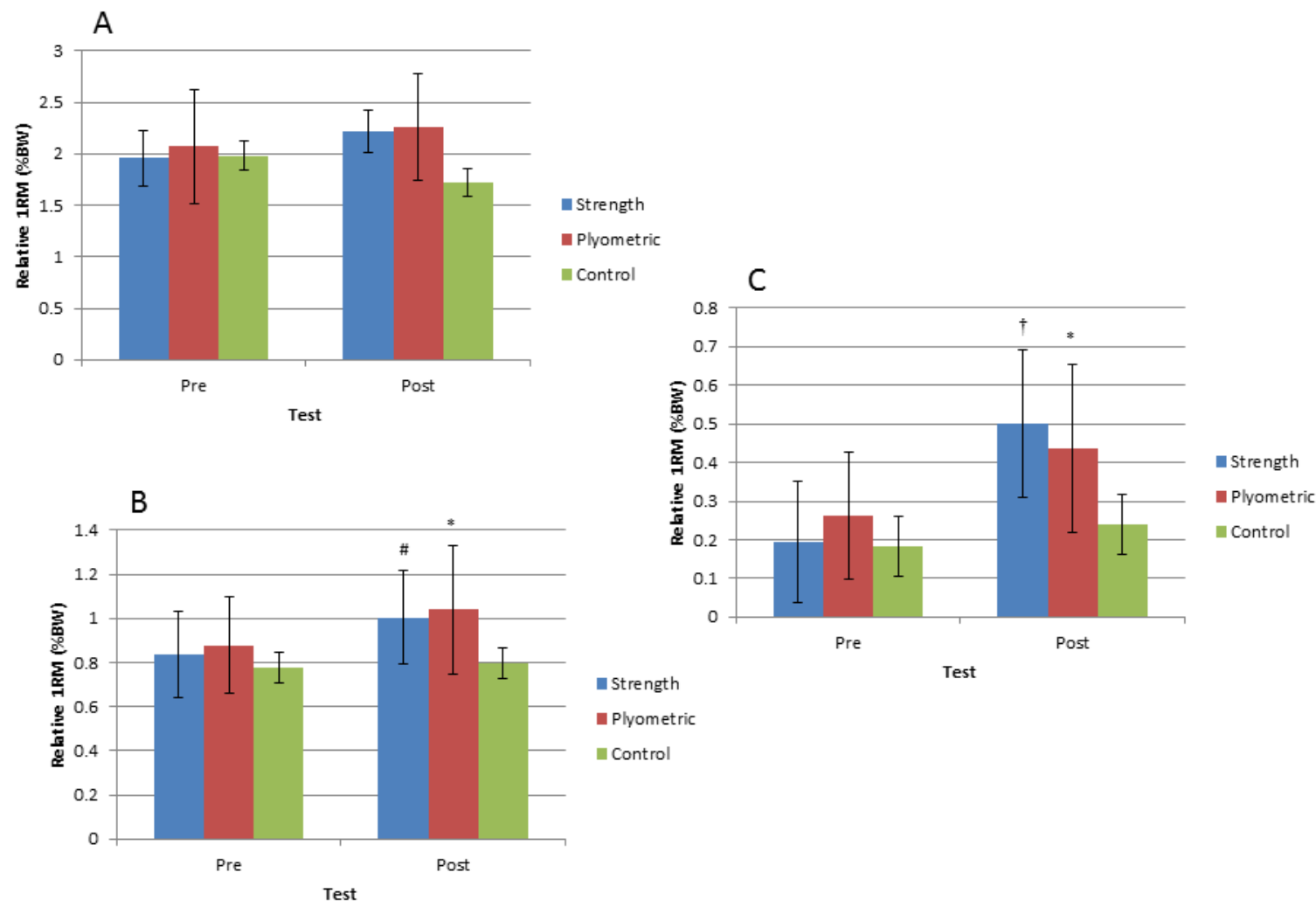

Figure 4.4 Mean Estimated 1RM Relative to Body Mass. A. Leg Press, B. Leg Extension \#Significantly different from CG $(p=0.009) *$ Significantly different from CG ( $p=0.007)$, C. Single Leg Lunge 'Significantly different from PG and CG ( $<$ $0.05)$ * *Significantly different from CG $(p<0.05)$.

\section{Isokinetic Measures}

Peak knee joint torque was calculated relative to body mass in both flexion and extension at $60^{\circ} / \mathrm{sec}, 120 \% \mathrm{sec}$, and $180^{\circ} / \mathrm{sec}$ (Table 4.2). There was a pattern for PG to have higher relative peak torque during knee flexion and SG being higher in knee extension at all velocities. Of the three velocities in both directions, there were only two 
significant group $\mathrm{x}$ time effects: $60 \%$ sec extension $(\mathrm{p}<0.001)$, and $180 \%$ sec flexion $(\mathrm{p}=$ 0.028). Post-hoc analysis determined that both PG $(\mathrm{p}=0.002)$ and SG $(\mathrm{p}=0.001)$ were significantly higher than CG while no difference was found between PG and SG at $60^{\circ} / \mathrm{sec}$ extension. PG was significantly higher than SG at $180^{\circ} / \mathrm{sec}$ flexion $(\mathrm{p}=0.025)$ and had no difference from CG $(\mathrm{p}>0.05)$. There were no significant differences between groups at $60^{\circ} / \mathrm{sec}$ flexion, $120 \% \mathrm{sec}$ flexion and extension, or $180^{\circ} / \mathrm{sec}$ extension $(\mathrm{p}>$ $0.05)$.

Average knee joint power was calculated relative to body mass in both flexion and extension at $60 \% \mathrm{sec}, 120 \% \mathrm{sec}$, and $180 \% \mathrm{sec}$ (Table 4.3 ). There were significant group $\mathrm{x}$ time interactions at all 3 velocities in both extension and flexion $(\mathrm{p}<0.001)$. At $60 \%$ sec flexion, PG was significantly more powerful than SG and CG ( $\mathrm{p}<0.001)$, and no difference between SG and CG ( $\mathrm{p}>0.05)$. However, at 60\% $/ \mathrm{sec}$ extension, $\mathrm{SG}$ was significantly higher than PG $(\mathrm{p}=0.002)$ and $\mathrm{CG}(\mathrm{p}=0.001)$, while there was no difference between PG and CG ( $\mathrm{p}>0.05)$. At $120^{\circ} / \mathrm{sec}, \mathrm{PG}$ was significantly more powerful than SG $(\mathrm{p}<0.001)$ and CG $(\mathrm{p}=0.002)$ and no difference between SG and CG ( $p>0.05)$ during knee flexion. PG was also significantly more powerful during knee extension than SG and CG ( $p<0.001)$ with no difference between $S G$ and CG $(p>0.05)$. Similarly, at $180^{\circ} / \mathrm{sec}, \mathrm{PG}$ was significantly higher than SG and CG $(\mathrm{p}<0.005)$ during knee flexion while SG and CG did not differ. PG was also significantly higher during knee extension compared to SG $(\mathrm{p}=0.001)$ and CG $(\mathrm{p}<0.001)$ with no difference between SG and CG. 
Table 4.2 Peak Knee Torque at Pre and Post-Testing During 8 Weeks of Resistance Training or Plyometric Training in Older Adults

\begin{tabular}{|c|c|c|c|c|c|}
\hline \multirow[b]{2}{*}{ Peak Knee Torque } & \multirow[b]{2}{*}{ Group } & \multirow[b]{2}{*}{ Pre } & \multirow[b]{2}{*}{ Post } & \multicolumn{2}{|c|}{$p$ Value* } \\
\hline & & & & Group x Time & Comparison $\dagger$ \\
\hline \multirow[t]{3}{*}{$60^{\circ} / \mathrm{sec}$ Flexion } & SG & $0.86 \pm 0.24$ & $0.96 \pm 0.24$ & 0.285 & \\
\hline & PG & $1.06 \pm 0.24$ & $1.18 \pm 0.25$ & & \\
\hline & $\mathrm{CG}$ & $0.97 \pm 0.13$ & $1.01 \pm 0.10$ & & \\
\hline \multirow[t]{3}{*}{$60^{\circ} / \mathrm{sec}$ Extension } & $\mathrm{SG}$ & $1.33 \pm 0.33$ & $1.51 \pm 0.41$ & $<0.001$ & $\mathrm{SG}>\mathrm{CG}$ \\
\hline & PG & $1.22 \pm 0.24$ & $1.39 \pm 0.28$ & & $\mathrm{PG}>\mathrm{CG}$ \\
\hline & CG & $1.19 \pm 0.22$ & $1.15 \pm 0.20$ & & \\
\hline \multirow[t]{3}{*}{$120^{\circ} / \mathrm{sec}$ Flexion } & SG & $0.82 \pm 0.21$ & $0.84 \pm 0.19$ & 0.08 & \\
\hline & PG & $0.95 \pm 0.19$ & $1.06 \pm 0.23$ & & \\
\hline & CG & $0.84 \pm 0.16$ & $0.86 \pm 0.14$ & & \\
\hline \multirow[t]{3}{*}{$120^{\circ} / \mathrm{sec}$ Extension } & SG & $1.05 \pm 0.28$ & $1.17 \pm 0.31$ & 0.22 & \\
\hline & PG & $0.97 \pm 0.26$ & $1.09 \pm 0.21$ & & \\
\hline & $\mathrm{CG}$ & $0.89 \pm 0.14$ & $0.93 \pm 0.15$ & & \\
\hline \multirow[t]{3}{*}{$180^{\circ} / \mathrm{sec}$ Flexion } & $\mathrm{SG}$ & $0.81 \pm 0.16$ & $0.76 \pm 0.23$ & 0.03 & \\
\hline & PG & $0.87 \pm 0.16$ & $0.95 \pm 0.25$ & & $\mathrm{PG}>\mathrm{SG}$ \\
\hline & $\mathrm{CG}$ & $0.79 \pm 0.13$ & $0.79 \pm 0.12$ & & \\
\hline \multirow[t]{3}{*}{$180^{\circ} / \mathrm{sec}$ Extension } & SG & $0.92 \pm 0.25$ & $1.00 \pm 0.31$ & 0.24 & \\
\hline & PG & $0.84 \pm 0.25$ & $0.98 \pm 0.22$ & & \\
\hline & $\mathrm{CG}$ & $0.76 \pm 0.16$ & $0.77 \pm 0.16$ & & \\
\hline
\end{tabular}

Values shown are mean \pm SD.

*Repeated measures ANOVA

\#Isokinetic relative to body mass in $\mathrm{N} \cdot \mathrm{m}$

$\dagger$ Within-group multiple comparisons for pre and post test

$\mathrm{SG}=$ strength group; $\mathrm{PG}=$ plyometric group; $\mathrm{CG}=$ control group 
Table 4.3 Average Knee Power at Pre and Post-Testing During 8 Weeks of Resistance Training or Plyometric Training in Older Adults

\begin{tabular}{cc|cccc}
\hline & & & & \multicolumn{2}{c}{$p$ Value* } \\
\cline { 3 - 6 } Average Power & Group & Pre & Post & Group x Time & Comparison' \\
\hline $60^{\circ} /$ sec Flexion & SG & $0.98 \pm 0.24$ & $0.64 \pm 0.18$ & $<0.001$ & \\
& PG & $0.90 \pm 0.18$ & $1.12 \pm 0.28$ & & PG > SG, CG \\
& CG & $0.88 \pm 0.16$ & $0.70 \pm 0.11$ & & \\
\hline $60^{\circ} /$ sec Extension & SG & $0.56 \pm 0.18$ & $1.49 \pm 0.18$ & $<0.001$ & SG > PG, CG \\
& PG & $0.75 \pm 0.15$ & $1.25 \pm 0.28$ & & \\
& CG & $0.65 \pm 0.11$ & $0.70 \pm 0.11$ & & \\
\hline $120^{\circ} /$ sec Flexion & SG & $1.55 \pm 0.43$ & $1.00 \pm 0.27$ & $<0.001$ & \\
& PG & $1.27 \pm 0.14$ & $1.99 \pm 0.49$ & & PG > SG, CG \\
& CG & $1.27 \pm 0.13$ & $1.06 \pm 0.30$ & & \\
\hline $120^{\circ} /$ sec Extension & SG & $1.96 \pm 0.48$ & $1.37 \pm 0.16$ & $<0.001$ & PG > SG, CG \\
& PG & $1.29 \pm 0.22$ & $1.90 \pm 0.38$ & & \\
\hline $180^{\circ} /$ sec Flexion & CG & $1.37 \pm 0.26$ & $1.05 \pm 0.17$ & & \\
& SG & $1.57 \pm 0.30$ & $1.18 \pm 0.37$ & $<0.001$ & PG > SG, CG \\
& PG & $2.13 \pm 0.40$ & $2.54 \pm 0.57$ & & \\
\hline CG & $1.25 \pm 0.42$ & $1.19 \pm 0.39$ & & \\
\hline $180^{\circ} /$ sec Extension & SG & $1.38 \pm 0.43$ & $1.29 \pm 0.21$ & $<0.001$ & PG > SG, CG \\
& PG & $1.47 \pm 0.43$ & $2.29 \pm 0.44$ & & \\
\hline & CG & $1.16 \pm 0.33$ & $0.95 \pm 0.15$ & & \\
\hline
\end{tabular}

Values shown are mean \pm SD.

*Repeated measures ANOVA

\#Isokinetic relative to body mass in watts

$\dagger$ Within-group multiple comparisons for pre and post test

$\mathrm{SG}=$ strength group $; \mathrm{PG}=$ plyometric group; $\mathrm{CG}=$ control group 


\section{CHAPTER 5: DISCUSSION}

As humans age into the $5^{\text {th }}$ and $6^{\text {th }}$ decade, muscular strength has been shown to decrease by up 1-2\% per year, while muscular power decreases at an even higher percentage (approximately 3-4\% per year). ${ }^{3}$ Because of this rapid decrease of muscular power and its subsequent negative impact on health outcomes in older adults, it is important to explore exercise options to reduce the typical decline in muscular power with aging. Therefore, the purpose of this study was to determine if performing plyometrics in an AlterG treadmill would lead to improved power outputs and functional measurements in older adults when compared to traditional resistance training. The results of this study indicated that performing plyometric exercises led to improvements in a chair sit-to-stand task, a timed stair climb, estimated 1RM for three resistance exercises, and several isokinetic measures. Moreover, the PG significantly improved several of these measures compared to a control group while completing less total workload than the SG, which has been seen in previous studies. ${ }^{13,36,37}$ To the author's knowledge, this is the first plyometric-specific training program studied in older adults, as well as using a weight supported treadmill for the utilization of the power exercises.

The PG was shown to significantly improve their functional strength in both the chair sit-to-stand and stair climb. In the chair stand, the PG improved by $21.7 \%$ while the SG improved by $12.5 \%$. PG was significantly greater than the CG $(\mathrm{p}=0.013)$, with no statistical difference between SG and CG. The $21.7 \%$ improvement is nearly double previous findings of high-velocity power resistance training in which improvements of 10.4-12\% have been reported. ${ }^{37,57,58}$ The results of this study found that the PG improved 
the timed stair climb by $14.8 \%$ while SG improved it by $7.4 \%$. This was significantly greater than the CG, whereas the SG was not significantly different than CG. Strength training alone has been shown to improve stair climb time. Capodaglio et al. found a $12 \%$ improvement in their time to climb stairs compared to control after one year of performing only two lower body exercises 3 times per week for 1 year. ${ }^{53}$ However, when comparing strength training to power training (or high velocity training), the power group has greater improvements than strength training groups in functional measures such as the time to climb stairs compared to controls. For example, a group training using maximal movement velocity significantly improved their stair climb time after 8 weeks of training compared to control whereas the strength group performing slower velocity movements did not.${ }^{37}$ The present study supports several previous findings that highvelocity resistance training can improve functional ability in older adults. ${ }^{37,57,58}$

The PG was also able to increase their 1RM in the leg press but not significantly, but did significantly increase in the leg extension and single leg lunge compared to CG. Interestingly, there was no difference in strength gains between PG and SG in the leg press or leg extension. SG did improve their single leg lunge compared to PG, likely due to a training effect of the exercise as it was a new lift for many participants. Previous studies have demonstrated the effects of plyometrics and strength gains. It has been postulated that the strength gains elicited through plyometrics are likely due to enhanced neural adaptations, a greater maximal rate of force development. ${ }^{60,62}$ In one study of young adults, plyometric training increased leg press $1 \mathrm{RM}$ by nearly $20 \% .{ }^{76}$ Results of the present study demonstrated an average increase of $9.1 \%$. In the leg extension, PG (p $=0.009)$ and $\mathrm{SG}(\mathrm{p}=0.007)$ were both significantly greater than $\mathrm{CG}$, with no differences 
between each other $(\mathrm{p}>0.05)$. The strength results of the present study is in agreement with previous studies that described plyometric training may enhance the ability to rapidly develop force, therefore allowing for greater improvements in maximal rate of force development. ${ }^{60}$ The strength gains seen in the SG in the present study are consistent with the results reported in previous studies. Previously, it has been shown that both power training and strength training groups can exhibit similar strength gains. One study showed overall muscle strength increases of about $50 \%$ for both power and strength training groups. ${ }^{37}$ This indicates that that power training groups are receiving similar improvements in strength as their strength training counterparts. However, it has been shown in previous studies as well as the current study that they are able to increase to a greater extent, ${ }^{37,38,57}$ and with more functional improvements while performing less total work. Less total work per exercise can be explained by comparing one subject from each exercise group. For example, a participant that is performing $3 \times 10$ on the leg press using $110 \mathrm{~kg}$ of resistance will lift a sum total of $3300 \mathrm{~kg}$ for that exercise. In comparison, a participant that weighs $80 \mathrm{~kg}$ and is performing $3 \times 10$ squat jumps in the AlterG at $80 \%$ of their body weight, they will lift a total of $1920 \mathrm{~kg}$. While this calculation may be an oversimplification of the total work done during a plyometric exercise, it gives a better understanding of the lower amount of work being performed during a plyometric exercise versus a traditional resistance training exercise.

Previous research has shown that resistance training for power in older adults can lead to improvements in functional abilities ${ }^{11,17,56}$ and an increase in muscular strength, size, and power. ${ }^{3,18,32,36,61}$ Another approach to power training are plyometric exercises. These exercises have also previously demonstrated an increase power output in a variety 
of forms such as vertical jump, sprint speed, and agility but have been primarily researched in children and college-aged adults. ${ }^{26,59,65,76}$ To the authors' knowledge, this is the first study that employed a plyometric specific training program for older adults. This novel study incorporated plyometrics in an older adult population through use of a weight supported treadmill and found similar results in functional measures, strength gains, and power output that have been recognized in young, healthy adults. For example, in a recent meta-analysis on plyometric training and its effect on muscular strength, it supported several previous studies that plyometrics can increase muscular strength, by up to $20 \mathrm{~kg}$ in young, healthy adults. ${ }^{60}$ The strength results in this study appear similar to previous findings with an average increase of $12 \mathrm{~kg}, 9 \mathrm{~kg}$, and $12 \mathrm{~kg}$ in the leg press, leg extension, and single leg lunge, respectively. This is noteworthy because the group participating in strength training did not differ significantly from the plyometric group who performed less absolute work per session, which is also in agreement with previous findings that power training can attain similar strength gains while performing less absolute work. ${ }^{37,56}$

Another key finding was the impact on average knee extension and flexion power output during isokinetic tests. The PG was significantly more powerful than SG and CG at all 3 velocities in both flexion and extension except for $60^{\circ} / \mathrm{sec}$ extension. After 8 weeks of plyometric training, the PG demonstrated a significantly higher power output in knee flexion at $60 \% \mathrm{sec}, 120 \% \mathrm{sec}$, and $180 \%$ sec and knee extension at $120 \% \mathrm{sec}$ and $180^{\circ} / \mathrm{sec}$ compared to the SG and CG. This is likely attributed to the rapid knee flexion that occurs during the plyometric movement, whereas the SG typically completes their exercise movement in a slower manner. Specificity of training should also be considered 
when interpreting the results from this study. During their training program, the SG performed exercises at a slower velocity than the PG. This specificity of training was demonstrated in the results at the lower velocity isokinetic measures, namely the $60^{\circ} / \mathrm{sec}$ extension. During the $60^{\circ} / \mathrm{sec}$ extension test, the SG significantly improved their power by $166 \%$, compared to the PG who improved by $66 \%(\mathrm{p}<0.001)$. However, during their training program, the PG typically performed exercises at a higher velocity. While the exact velocity of movement was not measured during the training, SG performed the movement in $\sim 2-3$ seconds in both concentric and eccentric motion while the explosive jumping movement that the PG performed was much quicker. This specificity of training was demonstrated by greater improvements in the moderate and higher velocity measures. For example, the SG was significantly greater at post testing at $60 \% \mathrm{~s}$ in both knee extension power and peak torque $(\mathrm{p}<0.001)$. Because most human movement occurs at velocities that are higher than the movement of traditional strength training, this may be one possibility to explain why performing higher-velocity and power-type movements during exercise training leads to greater functional outcomes.

The results from the present study indicate that older adults performing plyometrics in a weight supported treadmill can significantly improve knee extensor and flexor power output to a greater extent than participating in traditional resistance training. While muscular activity was not directly measured in this study, one likely hypothesis for the increase in strength and power is due to the neuromuscular adaptations the participants likely gained. Because the participants had no previous resistance training experience, the initial strength gains were likely due to enhanced neural pathways which have been shown to be attainable in older adults in the same manner as young 
people. ${ }^{33,54,77}$ It is widely accepted that the majority of strength increases during the initial weeks of training are due to neural adaptations such as increased neural drive and increased muscular activity of the agonist muscles. ${ }^{33,61,77,78}$ Furthermore, these neural adaptations have been shown to be similar in magnitude in older adults. ${ }^{78}$ The improvements in strength are important for the older population whether due to enhanced neural adaptations or the combination of both neural and muscle changes. Because it was shown that improvements in strength also translated into other functional measures such as stair climbing speed and improving sit-to-stand time, these are beneficial because they may presumably lead to an improved quality of life and extending independence.

As stated previously, this is the first study that used plyometric specific training in older adults as well as the first training specific study utilizing older adults in the AlterG. This was made possible due to performing such exercises in a weight supported treadmill that allows for added safety of the participant through stabilization and decreasing body weight. There were no adverse events in either of the groups during training sessions, supporting the safety of performing plyometrics in the AlterG. Although anecdotal, participants in the PG typically had a rating of perceived exertion (RPE) lower than the SG. In addition, delayed onset muscle soreness (DOMS) was rarely mentioned by participants in the PG whereas the SG commonly stated the feeling of DOMS. Decreased perceived effort and lower incidences of DOMS with plyometric training are both factors that would likely improve exercise compliance in previously untrained older adults.

While the overload principle for resistance training is well known, implementing an overload in the PG has not yet been established in a weight supported treadmill. In this 
study, we typically started the participants at a workload of around of $75 \%$ body mass and increased when the participant reported that performing the exercises became "too easy". While there are no current guidelines for the intensities at which exercise occurs in the AlterG, the current study used the RPE scale (1-10; 1 being extremely light and 10 being maximal exertion) after the final set of each exercise. Once the participant could successfully complete 3 sets of 10 reps with an RPE of 7-8 (very hard) the bodyweight increased by $1 \%$. Average progression for the leg press and the squat jump are show in in Appendix H. The rate of progression between both groups was quite similar. Future work is needed to further clarify the appropriate intensity of plyometric training for older or deconditioned populations using a weight supported treadmill.

Strengths of the study include a high rate of compliance. On average, participants completed 21/23 training visits and no participant missed a testing visit. Another strength of the study is the use of the AlterG treadmill. This piece of equipment allowed for older adults to complete plyometric exercises safely and effectively in a manner that they typically would not have been able to according to NSCA guidelines. Finally, measures of strength, functional strength, and power calculated from an isokinetic dynamometer allow for both clinical and practical applications of the study findings.

There are limitations that may have impacted the results of the study. First, a relatively small sample size was used, with only 3 males participating. Another limitation was the length of the study, however previous training studies have found significant changes in strength and functional measures in 8 weeks. ${ }^{48,49}$ Previously noted, neural adaptations are the primary cause of strength gains during the initial start of training. It is unlikely that there were significant changes at the muscular level for an 8 week training 
study. Increasing the length of study should be investigated in the future, and measures of muscular level adaptations such as changes in cross sectional area or muscle fiber composition could be studied. Finally, weight supported treadmills are often costly and rarely found outside of research and rehab facilities.

\section{Future Research}

Future research should investigate other types of plyometric exercises to be performed in the AlterG treadmill. The choice of squat jumps, single leg jumps, and power skips were to be as comparable to the resistance training exercises of leg press, single leg lunge, and leg extension as well as the relatively straight forward movement patterns. It would also be beneficial to determine optimal volume and foot contacts when performing plyometrics in the AlterG. Other research may focus on developing an overload principle for people exercising in the AlterG.

\section{Conclusions}

The results of this study indicate that older adults performing 8 weeks of plyometrics in a weight supported treadmill can significantly improve the time to climb a flight of stairs, muscular strength in the leg press, leg extension, single leg lunge, and isokinetic knee flexor and extensor power. These significant changes were accomplished through performing less total absolute work than the strength training group, meaning the total amount of weight moved each exercise session was much lower in the treadmill compared to the resistance exercises. Practical applications of these findings include the potential for new exercise prescription for older adults. Many of the participants completed a majority of their exercises at $80-85 \%$ of their body weight, demonstrating the possibility of revisiting the guidelines set forth by the National Strength and 
Conditioning Association. Based on the results of the study, older adults may benefit from performing a modified plyometric exercise program through a weight supported treadmill. These benefits include increasing their functional abilities, lower body muscular strength, and power. This was the first study that employed a plyometric training program in older adults. Additional research is recommended to investigate optimal frequency, intensity, type, and time for these exercises to be utilized by older adults. 


\section{REFERENCES}

1. Suzman R, Beard J. Global Health and Aging: National Institute of Health; 2011.

2. Colby S, Ortman J. TheBaby Boom Cohort in the United States: 2012 to 2060. Current Population Reports. Washington, DC.: U.S. Census Bureau; 2014.

3. Skelton DA, Greig CA, Davies JM, Young A. Strength, power and related functional ability of healthy people aged 65-89 years. Age Ageing. 1994;23(5):371-377.

4. Pescatello L, Arena R, Riebe D, Thompson P. ACSM's Guidelines for Exercise Testing and Prescription. 9th ed. Baltimore, MD: Wolters Kluwer Health; 2014.

5. Goodpaster BH, Park SW, Harris TB, et al. The loss of skeletal muscle strength, mass, and quality in older adults: the health, aging and body composition study. $\mathrm{J}$ Gerontol A Biol Sci Med Sci. 2006;61(10):1059-1064.

6. Lexell J, Taylor CC, Sjöström M. What is the cause of the ageing atrophy? Total number, size and proportion of different fiber types studied in whole vastus lateralis muscle from 15- to 83-year-old men. J Neurol Sci. 1988;84(2-3):275294.

7. Roubenoff R, Hughes VA. Sarcopenia: current concepts. J Gerontol A Biol Sci Med Sci. 2000;55(12):M716-724.

8. Goodpaster BH, Carlson CL, Visser M, et al. Attenuation of skeletal muscle and strength in the elderly: The Health ABC Study. J Appl Physiol (1985). 2001;90(6):2157-2165.

9. Penninx BW, Messier SP, Rejeski WJ, et al. Physical exercise and the prevention of disability in activities of daily living in older persons with osteoarthritis. Arch Intern Med. 2001;161(19):2309-2316.

10. Lawton MP, Brody EM. Assessment of older people: self-maintaining and instrumental activities of daily living. Gerontologist. 1969;9(3):179-186.

11. Hruda KV, Hicks AL, McCartney N. Training for muscle power in older adults: effects on functional abilities. Can J Appl Physiol. 2003;28(2):178-189.

12. Sherrington C, Whitney JC, Lord SR, Herbert RD, Cumming RG, Close JC. Effective exercise for the prevention of falls: a systematic review and metaanalysis. J Am Geriatr Soc. 2008;56(12):2234-2243. 
13. Orr R, de Vos NJ, Singh NA, Ross DA, Stavrinos TM, Fiatarone-Singh MA. Power training improves balance in healthy older adults. J Gerontol A Biol Sci Med Sci. 2006;61(1):78-85.

14. Sleet D, Moffett D, Stevens J. CDC's research portfolio in older adult fall prevention: a review of progress, 1985-2005, and future research directions. Journal of Safety Research. 2006;39(3):259-267.

15. Carter ND, Kannus P, Khan KM. Exercise in the prevention of falls in older people: a systematic literature review examining the rationale and the evidence. Sports Med. 2001;31(6):427-438.

16. Stevens JA, Corso PS, Finkelstein EA, Miller TR. The costs of fatal and non-fatal falls among older adults. Inj Prev. 2006;12(5):290-295.

17. Bottaro M, Machado SN, Nogueira W, Scales R, Veloso J. Effect of high versus low-velocity resistance training on muscular fitness and functional performance in older men. Eur J Appl Physiol. 2007;99(3):257-264.

18. Earles DR, Judge JO, Gunnarsson OT. Velocity training induces power-specific adaptations in highly functioning older adults. Arch Phys Med Rehabil. 2001;82(7):872-878.

19. Hess JA, Woollacott M. Effect of high-intensity strength-training on functional measures of balance ability in balance-impaired older adults. J Manipulative Physiol Ther. 2005;28(8):582-590.

20. Toraman NF, Erman A, Agyar E. Effects of multicomponent training on functional fitness in older adults. J Aging Phys Act. 2004;12(4):538-553.

21. Bean JF, Kiely DK, Herman S, et al. The relationship between leg power and physical performance in mobility-limited older people. J Am Geriatr Soc. 2002;50(3):461-467.

22. Porter MM. Power training for older adults. Appl Physiol Nutr Metab. 2006;31(2):87-94.

23. Association NSAC. Essentials of Strength Training and Conditioning. 4 ed. Champaign, IL: Human Kinetics; 2016.

24. Cormie P, McBride JM, McCaulley GO. Power-time, force-time, and velocitytime curve analysis during the jump squat: impact of load. J Appl Biomech. 2008;24(2):112-120.

25. Barnes JL, Schilling BK, Falvo MJ, Weiss LW, Creasy AK, Fry AC. Relationship of jumping and agility performance in female volleyball athletes. J Strength Cond Res. 2007;21(4):1192-1196. 
26. Miller MG, Herniman JJ, Ricard MD, Cheatham CC, Michael TJ. The effects of a 6-week plyometric training program on agility. J Sports Sci Med. 2006;5(3):459465.

27. Luebbers PE, Potteiger JA, Hulver MW, Thyfault JP, Carper MJ, Lockwood RH. Effects of plyometric training and recovery on vertical jump performance and anaerobic power. J Strength Cond Res. 2003;17(4):704-709.

28. Markovic G. Does plyometric training improve vertical jump height? A metaanalytical review. Br J Sports Med. 2007;41(6):349-355; discussion 355.

29. Figueroa M, Manning J, Escamilla P. Physiological responses to the AlterG antigravity treadmill. International Journal of Applied Science and Technology. 2011;1(6):92-97.

30. McNeill DK, Kline JR, de Heer HD, Coast JR. Oxygen consumption of elite distance runners on an anti-gravity treadmill@. J Sports Sci Med. 2015;14(2):333339.

31. Patil S, Steklov N, Bugbee WD, Goldberg T, Colwell CW, D'Lima DD. Antigravity treadmills are effective in reducing knee forces. J Orthop Res. 2013;31(5):672-679.

32. Hunter GR, McCarthy JP, Bamman MM. Effects of resistance training on older adults. Sports Med. 2004;34(5):329-348.

33. Aagaard P, Simonsen EB, Andersen JL, Magnusson P, Dyhre-Poulsen P. Increased rate of force development and neural drive of human skeletal muscle following resistance training. J Appl Physiol (1985). 2002;93(4):1318-1326.

34. Runge M, Rittweger J, Russo CR, Schiessl H, Felsenberg D. Is muscle power output a key factor in the age-related decline in physical performance? A comparison of muscle cross section, chair-rising test and jumping power. Clin Physiol Funct Imaging. 2004;24(6):335-340.

35. Martin JC, Farrar RP, Wagner BM, Spirduso WW. Maximal power across the lifespan. J Gerontol A Biol Sci Med Sci. 2000;55(6):M311-316.

36. de Vos NJ, Singh NA, Ross DA, Stavrinos TM, Orr R, Fiatarone Singh MA. Optimal load for increasing muscle power during explosive resistance training in older adults. J Gerontol A Biol Sci Med Sci. 2005;60(5):638-647.

37. Henwood TR, Riek S, Taaffe DR. Strength versus muscle power-specific resistance training in community-dwelling older adults. J Gerontol A Biol Sci Med Sci. 2008;63(1):83-91. 
38. Skelton DA, Young A, Greig CA, Malbut KE. Effects of resistance training on strength, power, and selected functional abilities of women aged 75 and older. $J$ Am Geriatr Soc. 1995;43(10):1081-1087.

39. Tracy BL, Ivey FM, Hurlbut D, et al. Muscle quality. II. Effects Of strength training in 65- to 75-yr-old men and women. J Appl Physiol (1985). 1999;86(1):195-201.

40. McCartney N, Hicks AL, Martin J, Webber CE. A longitudinal trial of weight training in the elderly: continued improvements in year 2. J Gerontol A Biol Sci Med Sci. 1996;51(6):B425-433.

41. Frontera WR, Meredith CN, O'Reilly KP, Knuttgen HG, Evans WJ. Strength conditioning in older men: skeletal muscle hypertrophy and improved function. $\mathrm{J}$ Appl Physiol (1985). 1988;64(3):1038-1044.

42. Evans WJ. Exercise strategies should be designed to increase muscle power. J Gerontol A Biol Sci Med Sci. 2000;55(6):M309-310.

43. Newton RU, Murphy AJ, Humphries BJ, Wilson GJ, Kraemer WJ, Häkkinen K. Influence of load and stretch shortening cycle on the kinematics, kinetics and muscle activation that occurs during explosive upper-body movements. Eur J Appl Physiol Occup Physiol. 1997;75(4):333-342.

44. McNeely E. Introduction to plyometrics: Converting strength to power. NSCA's Performance Training Journal. 2005;6(5):19-22.

45. Peterson MD, Rhea MR, Sen A, Gordon PM. Resistance exercise for muscular strength in older adults: a meta-analysis. Ageing Res Rev. 2010;9(3):226-237.

46. Latham NK, Bennett DA, Stretton CM, Anderson CS. Systematic review of progressive resistance strength training in older adults. J Gerontol A Biol Sci Med Sci. 2004;59(1):48-61.

47. (CDC) CfDCaP. Self-reported falls and fall-related injuries among persons aged $\geq 65$ years--United States, 2006. MMWR Morb Mortal Wkly Rep. 2008;57(9):225-229.

48. Mihalko S, McAuley E. Strength training effects on subjective well-being and physical function in the elderly. Journal of Aging and Physical Activity. 1996;4:56-58.

49. Schlicht J, Camaione DN, Owen SV. Effect of intense strength training on standing balance, walking speed, and sit-to-stand performance in older adults. J Gerontol A Biol Sci Med Sci. 2001;56(5):M281-286.

50. Skelton D, McLaughlin A. Training functional ability in old age. Physiotherapy. 1996;82:159-167. 
51. Sherrington C, Lord SR. Home exercise to improve strength and walking velocity after hip fracture: a randomized controlled trial. Arch Phys Med Rehabil. 1997;78(2):208-212.

52. Skelton D, McLaughlin A. Training functional ability in old age. Physiotherapy. 1996;82(3):159-167.

53. Capodaglio P, Capodaglio Edda M, Facioli M, Saibene F. Long-term strength training for community-dwelling people over 75: impact on muscle function, functional ability and life style. Eur J Appl Physiol. 2007;100(5):535-542.

54. Aagaard P, Suetta C, Caserotti P, Magnusson SP, Kjaer M. Role of the nervous system in sarcopenia and muscle atrophy with aging: strength training as a countermeasure. Scand J Med Sci Sports. 2010;20(1):49-64.

55. Reid KF, Fielding RA. Skeletal muscle power: a critical determinant of physical functioning in older adults. Exerc Sport Sci Rev. 2012;40(1):4-12.

56. Miszko TA, Cress ME, Slade JM, Covey CJ, Agrawal SK, Doerr CE. Effect of strength and power training on physical function in community-dwelling older adults. J Gerontol A Biol Sci Med Sci. 2003;58(2):171-175.

57. Henwood TR, Taaffe DR. Improved physical performance in older adults undertaking a short-term programme of high-velocity resistance training. Gerontology. 2005;51(2):108-115.

58. Henwood TR, Taaffe DR. Short-term resistance training and the older adult: the effect of varied programmes for the enhancement of muscle strength and functional performance. Clin Physiol Funct Imaging. 2006;26(5):305-313.

59. Faigenbaum AD, McFarland JE, Keiper FB, et al. Effects of a short-term plyometric and resistance training program on fitness performance in boys age 12 to 15 years. J Sports Sci Med. 2007;6(4):519-525.

60. Sáez-Sáez de Villarreal E, Requena B, Newton RU. Does plyometric training improve strength performance? A meta-analysis. J Sci Med Sport. 2010;13(5):513-522.

61. Macaluso A, De Vito G. Muscle strength, power and adaptations to resistance training in older people. Eur J Appl Physiol. 2004;91(4):450-472.

62. Markovic G, Mikulic P. Neuro-musculoskeletal and performance adaptations to lower-extremity plyometric training. Sports Med. 2010;40(10):859-895.

63. Davies G, Riemann BL, Manske R. CURRENT CONCEPTS OF PLYOMETRIC EXERCISE. Int J Sports Phys Ther. 2015;10(6):760-786. 
64. Kato T, Terashima T, Yamashita T, Hatanaka Y, Honda A, Umemura Y. Effect of low-repetition jump training on bone mineral density in young women. J Appl Physiol (1985). 2006;100(3):839-843.

65. Fatouros I, Jamurtas A, Leontsini D, et al. Evaluation of plyometric exercise training, weight training, and their combination on vertical jumping performance and leg strength. The Journal of Strength \& Conditioning Research. 2000;14(4):470-476.

66. Bassey EJ, Rothwell MC, Littlewood JJ, Pye DW. Pre- and postmenopausal women have different bone mineral density responses to the same high-impact exercise. J Bone Miner Res. 1998;13(12):1805-1813.

67. Saxena A, Granot A. Use of an anti-gravity treadmill in the rehabilitation of the operated achilles tendon: a pilot study. J Foot Ankle Surg. 2011;50(5):558-561.

68. Tenforde AS, Watanabe LM, Moreno TJ, Fredericson M. Use of an antigravity treadmill for rehabilitation of a pelvic stress injury. PM R. 2012;4(8):629-631.

69. Guralnik JM, Simonsick EM, Ferrucci L, et al. A short physical performance battery assessing lower extremity function: association with self-reported disability and prediction of mortality and nursing home admission. J Gerontol. 1994;49(2):M85-94.

70. Lord SR, Murray SM, Chapman K, Munro B, Tiedemann A. Sit-to-stand performance depends on sensation, speed, balance, and psychological status in addition to strength in older people. J Gerontol A Biol Sci Med Sci. 2002;57(8):M539-543.

71. Bean JF, Kiely DK, LaRose S, Alian J, Frontera WR. Is stair climb power a clinically relevant measure of leg power impairments in at-risk older adults? Arch Phys Med Rehabil. 2007;88(5):604-609.

72. Drouin JM, Valovich-mcLeod TC, Shultz SJ, Gansneder BM, Perrin DH. Reliability and validity of the Biodex system 3 pro isokinetic dynamometer velocity, torque and position measurements. Eur J Appl Physiol. 2004;91(1):2229.

73. Hartmann A, Knols R, Murer K, de Bruin ED. Reproducibility of an isokinetic strength-testing protocol of the knee and ankle in older adults. Gerontology. 2009;55(3):259-268.

74. Reynolds JM, Gordon TJ, Robergs RA. Prediction of one repetition maximum strength from multiple repetition maximum testing and anthropometry. J Strength Cond Res. 2006;20(3):584-592.

75. Brzycki M. Strength test-predicting a one-rep max from reps-to-fatigue. Journal of Physical Education, Recreation \& Dance. 1993;64(1):88-90. 
76. de Villarreal ES, González-Badillo JJ, Izquierdo M. Low and moderate plyometric training frequency produces greater jumping and sprinting gains compared with high frequency. J Strength Cond Res. 2008;22(3):715-725.

77. Narici MV, Roi GS, Landoni L, Minetti AE, Cerretelli P. Changes in force, crosssectional area and neural activation during strength training and detraining of the human quadriceps. Eur J Appl Physiol Occup Physiol. 1989;59(4):310-319.

78. Häkkinen K, Alen M, Kallinen M, Newton RU, Kraemer WJ. Neuromuscular adaptation during prolonged strength training, detraining and re-strength-training in middle-aged and elderly people. Eur J Appl Physiol. 2000;83(1):51-62. 
APPENDIX A

Participant Recruitment Flyer 


\section{VOLUNTEERS NEEDED For an Exercise Training Study}

\section{Eligibility:}

1. 50 years or older

2. Have not participated in any resistance training the previous 6 months.

3. Have no history of joint replacements.

\section{What will I be doing?}

- You will be performing multiple pre and post tests involving strength, power, and body composition

- You will be exercising 3 times a week for 8 weeks

- Exercise sessions will last approximately 30-45 minutes

For more information call (605) 431-8619 or send an email to tylerdobbs@u.boisestate.edu

\begin{tabular}{|c|c|c|c|c|c|c|c|c|c|c|c|}
\hline 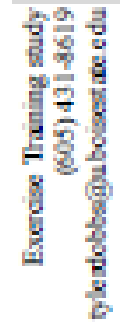 & 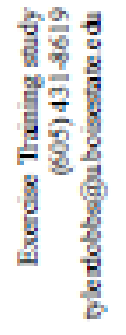 & 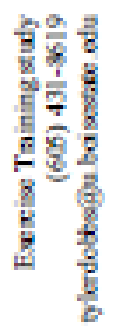 & 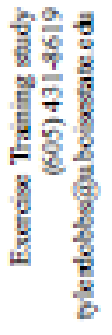 & 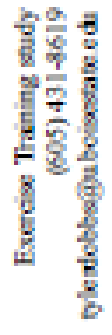 & 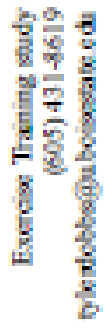 & 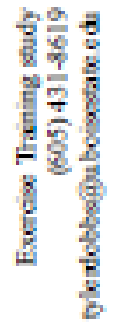 & 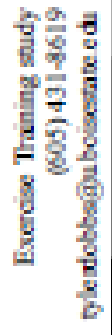 & 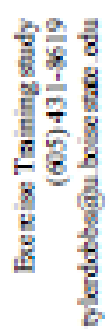 & 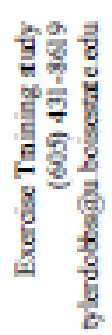 & 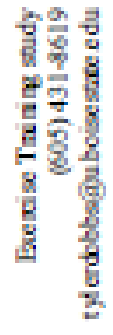 & 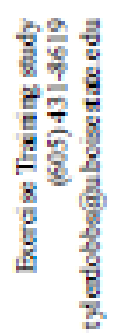 \\
\hline
\end{tabular}


APPENDIX B

Osher Recruitment Letter 
Hello,

My name is Tyler Dobbs. I am in my last semester of the Kinesiology graduate program at Boise State University, pursuing my masters in exercise physiology. I received my BS in exercise science from SDSU and began a position as an exercise physiologist in cardiac rehab for more than two years. I have a great amount of experience working with older adults in both the clinical and applied setting. Currently, I am the biomechanics graduate assistant as well as the GA leading the older adult strength training program held at BSU. I am also a certified exercise physiologist through the American College of Sports Medicine.

I am currently recruiting participants to enroll in my research study to complete my thesis. This study will be examining different exercise protocols in older adults and assessing various functional outcomes. The exercise sessions will take place in the brand new BSU Human Performance Lab and the Kinesiology gymnasium. This study will last for 8 weeks and you will be asked to exercise 3 times per week for around 30-45 minutes for the duration of the study. I am looking to get started as soon as possible. If you are interested in participating or have any questions about the study, I would be more than happy to answer them! Please email me at tylerdobbs@u.boisestate.edu or you can call me at 605-431-8619. Thank you for your continuing involvement in education and research at BSU! 
APPENDIX C

Health History Questionnaire 
Test Date:

\section{HEALTH HISTORY QUESTIONNAIRE}

NAME:

AGE: DATE OF BRTH:

Fint Last

ADDRESS:

Stret

City

Sato

$Z_{p}$

TELEPHONE:

E-mail address:

Person to contact in case of an emergency: Phone $\#$

(relationship)

Has your physician ever told you that you bave any of the following? (Yes or No)

YES NO If yes, explain:

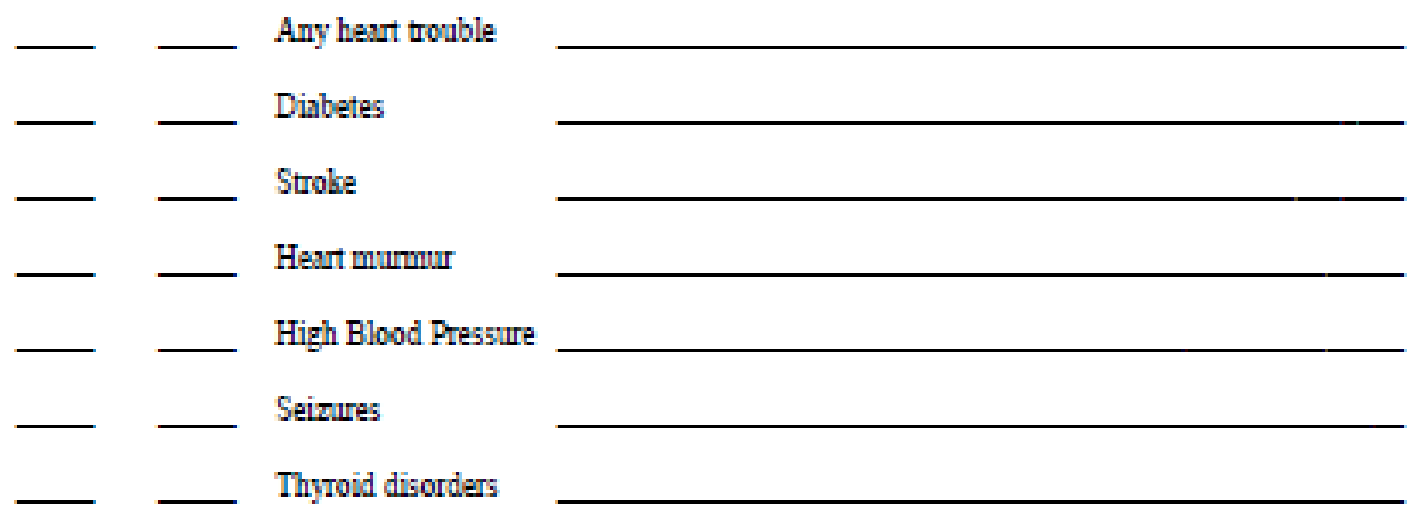

In the past 30 days, have you had any of the following? (Yes or No)

YES NO If yes, explain:

$-\quad$ Chest Pain
$-\quad$ Shortmess of breath 
In the past 30 days, have you had any of the following? (Yes or No)

YES NO

If yes, explain:

Feeling faint/dizzy

Heart palpitations

Severe Headache

Hospital admission

Are you taling any prescription or over-the counter medications? Yes _ No

Name of medication

Reason for Taking

For How Long?

Do you currently engage in resistance training activity on a regular basis? Yes No If so, what type? How many days per week?

How much time per session? (check one) $<15$ min $15-30 \mathrm{~min}$ $30-45 \mathrm{~min}$ $>60 \min$

How long have you been engaged in resistance training?

Do you ever have an uncomfortable shortmess of breath during exercise? Yes No

Do you ever have chest discomfort during exercise? Yes No

\section{FOR STAFF USE:}


APPENDIX D

Boise State University Institutional Review Board Approval Letter 


\section{BOISE STATE UNIVERSITY}

RESEARCH AND ECONOMIC DEVELOPMENT

Date: January 04, 2016

To: Tyler Dobbs

cc: Scott A. Conger

Shawn Simonson

From: Biomedical Institutional Review Board (MED-IRB)

c/o Office of Research Compliance

(ORC)

Subject: MED-IRB Notification of Approval - Original - 103-MED15-009

Increasing Power Output in Older Adults Utilizing Plyometrics in a Bodyweight

Supported

Treadmill

The Boise State University IRB has approved your protocol submission. Your protocol is in compliance with this institution's Federal Wide Assurance (\#0000097) and the DHHS Regulations for the Protection of Human Subjects (45 CFR 46).

Protocol

Number: 103-MED15-009 Expires: 1/3/2017
Received: 12/2/2015

Approved: 1/4/2016
Expedite

Review: d

Category: 4

Your approved protocol is effective until 1/3/2017. To remain open, your protocol must be renewed on an annual basis and cannot be renewed beyond 1/3/2019. For the activities to continue beyond 1/3/2019, a new protocol application must be submitted.

ORC will notify you of the protocol's upcoming expiration roughly 30 days prior to $1 / 3 / 2017$. You, as the PI, have the primary responsibility to ensure any forms are submitted in a timely manner for the approved activities to continue. If the protocol is not renewed before $1 / 3 / 2017$, the protocol will be closed. If you wish to continue the activities after the protocol is closed, you must submit a new protocol application for MED-IRB review and approval.

You must notify the MED-IRB of any additions or changes to your approved protocol using a Modification Form. The MED-IRB must review and approve the modifications before they can begin. When your activities are complete or discontinued, please submit a Final Report. An executive summary or other documents with the results of the research may be included.

All forms are available on the ORC website at http://goo.gl/D2FYTV

Please direct any questions or concerns to ORC at 426-5401 or humansubjects@boisestate.edu. 
Thank you and good luck with your research.

\section{Dr. Cheryl Jorcyk}

Chair

Boise State University Biomedical Institutional Review Board

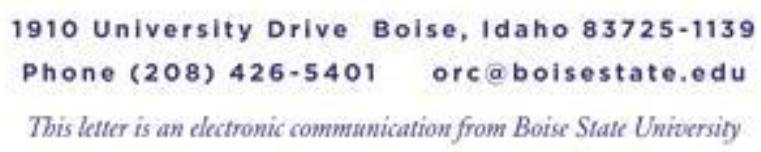


APPENDIX E

\section{Informed Consent Form}




\section{$\boldsymbol{B}$}

\section{BOISE STATE UNIVERSITY}

INFORMED CONSENT

Study Title: Increasing Power Output Utilizing Plyometrics in a Bodyweight Supported

Treadmill

Principal Investigator: Tyler Dobbs

Co-Investigators: Dr. Scott Conger, Dr.

Shawn Simonson

\section{Sponsor: N/A}

This consent form will give you the information you will need to understand why this research study is being done and why you are being invited to participate. It will also describe what you will need to do to participate as well as any known risks, inconveniences or discomforts that you may have while participating. We encourage you to ask questions at any time. If you decide to participate, you will be asked to sign this form and it will be a record of your agreement to participate. You will be given a copy of this form to keep.

\section{PURPOSE AND BACKGROUND}

The purpose of this research study is to compare two types of exercises and their benefits on functional ability. The two types of exercises used are traditional weight lifting exercises and plyometric exercises in a bodyweight supported treadmill (AlterG Treadmill). These kinds of exercises have both been shown to increase functional ability. To be in this study you must be 50 years or older and in good physical health with no history of resistance training in the previous 6 months.

\section{PROCEDURES}

This is an 8 week study in which you will exercise 3 times per week. The first visit will consist of paperwork and familiarization with the equipment. The second visit will involve pre-tests in which are described below. Visits 3-26 will consist of the exercise training. The final visit will consist of post-tests. If you are interested in the study, you will be given a physician consent to exercise form for your physician to review and sign.

\section{Visit 1 - Paperwork and testing familiarization (1.5hrs)}

During the first visit you will complete paperwork and be introduced to the exercise equipment. You will have an opportunity to perform the exercises with little or 
no weight to become familiar with them. You will also get to see how the AlterG treadmill works. Demonstration of the pre and post tests will also occur.

\section{Visit 2 - Pre-Tests (1.5 hrs)}

During the second visit you will perform 3 different tests in which your muscular power is measured. You will be demonstrated how the tests work and given an opportunity to practice. After a short warm up, you will perform a timed sit-to-stand, timed stair climb, and a knee extension/flexion test. The timed-sit-to stand test measures how quickly you can stand and sit from a chair. The timed stair climb will measure how quickly you can climb a flight of stairs consisting of 9 steps. Finally, the knee extension/flexion test will measure how much power you can generate by extending and flexing at the knee. This is measured by a piece of equipment that maintains a constant speed. A estimate of your one repetition maximum (1RM) will be also be measured by lifting as much weight as you can for up to 5 repetitions to determine the amount of weight that will be used during the exercise session and to also determine strength gains throughout the study. You will also have your body composition tested via a BodPod, in which you sit comfortably in a small pod momentarily and your body fat percentage is measured.

\section{Visits 3-26 (30-60 min)}

Visits 3-26 will consist of the exercise training visits. You will be asked to come 3 times per week for 8 weeks for a total of 24 training visits with at least 48 hours between visits. After performing a general warm-up, you will complete the exercises that are assigned to you. An experienced research personnel will be there at all times to answer any questions, and to make sure you are maintaining proper form and performing correct exercises. Using computer software, you will be randomly selected into one of three groups for the study. These groups are a strength training group (SG), plyometric training group (PG), or a control group (CG). SG will perform traditional strength training exercises using resistance training machines. PG will perform similar exercises but using a bodyweight supported treadmill. CG will be asked to not perform any exercises for the 8 weeks and to continue their normal activities.

Visit 12 or 4 weeks ( 1 hr)

At the halfway point of the study, you will be asked to perform the same pre-tests (except body composition) that you did in visit 2 to determine if you have become stronger and if you now have a new 1RM.

Visit 27 - Post Tests (1.5 hrs)

The last visit of the study will involve the same exact tests performed in visit 2 .

\section{RISKS}

As with any type of exercise there are potential risks that may occur while participating in this study. These may include acute muscle or joint injury, muscle/joint 
soreness, dizziness, nausea, and in more rare instances fainting and heart attack. The possibility of encountering a cardiac event is low in people with no previous history of cardiac disease. The Human Performance Laboratory has a planned response to an emergency and all testing personnel are CPR certified.

\section{BENEFITS}

There are no direct benefits from participating in this study. Benefits that you may encounter include an increase in muscle strength and size as well as an increased functional capacity such as performing activities of daily living.

\section{EXTENT OF CONFIDENTIALITY}

Reasonable efforts will be made to keep the personal information in your research record private and confidential. Any identifiable information obtained in connection with this study will remain confidential and will be disclosed only with your permission or as required by law. The members of the research team, and the Boise State University Office of Research Compliance (ORC) may access the data. The ORC monitors research studies to protect the rights and welfare of research participants. For this research project, the researchers are requesting demographic information. Due to the make-up of Idaho's population, the combined answers to these questions may make an individual person identifiable. The researchers will make every effort to protect your confidentiality. However, if you are uncomfortable answering any of these questions, you may leave them blank.

Your name will not be used in any written reports or publications which result from this research. Data will be kept for three years (per federal regulations) after the study is complete and then destroyed.

\section{PAYMENT}

You will not be paid for your participation in this study.

\section{PARTICIPATION IS VOLUNTARY}

You do not have to be in this study if you do not want to. You may also refuse to answer any questions you do not want to answer. If you volunteer to be in this study, you may withdraw from it at any time without consequences of any kind or loss of benefits to which you are otherwise entitled.

\section{QUESTIONS}

If you have any questions or concerns about your participation in this study, you may contact the Principal Investigator, Tyler Dobbs: 605-431-8619 or tylerdobbs@u.boisestate.edu. 
If you have questions about your rights as a research participant, you may contact the Boise State University Institutional Review Board (IRB), which is concerned with the protection of volunteers in research projects. You may reach the board office between 8:00 AM and 5:00 PM, Monday through Friday, by calling (208) 426-5401 or by writing: Institutional Review Board, Office of Research Compliance, Boise State University, 1910 University Dr., Boise, ID 83725-1138.

\section{DOCUMENTATION OF CONSENT}

I have read this form and decided that I will participate in the project described above. Its general purposes, the particulars of involvement and possible risks have been explained to my satisfaction. I understand I can withdraw at any time.

$\begin{array}{lll}\text { Printed Name of Study Participant } & \text { Signature of Study Participant } & \text { Date }\end{array}$ 
APPENDIX F

Physician Consent to Participate 
Increasing Power Output in Older Adults Utilizing a Weight Supported Treadmill Department of Kinesiology, Boise State University

\section{Health Provider Permission Information}

For your safety and as a precaution, the university requires that you obtain a consent to participate in this research study from your physician. This study involves strength and functional testing. The study lasts 8 weeks and you will be exercising 3 times per week for approximately 45-60 minutes.

The pre and post assessments will include:

- Strength testing, functional assessments, height and weight, and body composition

\section{Exercise training will include:}

- $\quad$ Lower body strength training

- $\quad$ Similar exercises in a weight supported treadmill

\section{How to obtain permission from your approved health care provider (M.D., P.A.,} N.P.)

1. You can call your health care provider or drop this form off and have them fax the information or this form to: 208-426-1894 Attention: Tyler Dobbs

2. Your provider may scan and email to: Tylerdobbs@u.boisestate.edu

3. You can also bring the signed form with you on your first session

4. If you or your provider has any questions they can contact:

Tyler Dobbs 605-431-8619 tylerdobbs@u.boisestate.edu 
Health Care Provider Name Provider Fax \#

Participants Name would like to obtain consent to participate in the strength and functional training research study that is described above. Yes, the above named person may participate.

Comments:

_ No, they may not participate

Signature

Please fax or email this form back to: 208-426-1894 Attention: Tyler Dobbs tylerdobbs@u.boisestate.edu 
APPENDIX G

Mean Normative Data 


\section{Strength Group Mean Normative Data}

Strength Group Mean Normative Data

\begin{tabular}{|c|c|c|c|}
\hline Variable & Pre & Mid & Post \\
\hline Age & 66.25 & 66.25 & 66.25 \\
\hline Height (cm) & 165.44 & 165.44 & 165.44 \\
\hline Weight (kg) & 80.12 & 80.62 & 80.39 \\
\hline Body_Fat $(\%)$ & 37.94 & & 38.84 \\
\hline BMI $\left(\mathrm{kg} / \mathrm{m}^{2}\right)$ & 29.27 & 29.46 & 29.44 \\
\hline Sit/Stand (s) & 8.25 & 7.72 & 7.22 \\
\hline Stair_Climb (s) & 3.32 & 3.12 & 3.07 \\
\hline $60^{\circ} /$ sec Torque Flexion & 67.76 & 76.10 & 75.38 \\
\hline $60^{\circ} /$ sec Torque Extension & 104.30 & 111.88 & 119.06 \\
\hline $60^{\circ} /$ sec Power Flexion & 44.21 & 50.91 & 50.23 \\
\hline $60^{\circ} /$ sec Power Extension & 62.76 & 66.92 & 75.18 \\
\hline $120^{\circ} / \mathrm{sec}$ Torque Flexion & 64.77 & 65.97 & 48.53 \\
\hline $120 \%$ sec Torque Extension & 81.52 & 86.10 & 91.62 \\
\hline 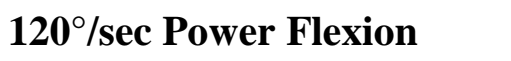 & 77.86 & 80.32 & 78.54 \\
\hline $120^{\circ} /$ sec Power Extension $^{2}$ & 94.71 & 99.08 & 109.89 \\
\hline $180^{\circ} /$ sec Torque Flexion & 63.71 & 60.69 & 59.16 \\
\hline $180^{\circ} /$ sec Torque Extension & 71.49 & 70.39 & 78.54 \\
\hline 180/sec Power Flexion & 100.63 & 96.89 & 92.60 \\
\hline $180^{\circ} /$ sec Power Extension $^{2}$ & 108.80 & 104.98 & 121.18 \\
\hline Leg Press 1RM (kg) & 154.55 & 167.99 & 175.33 \\
\hline Leg Extension 1RM (kg) & 65.44 & 73.21 & 78.46 \\
\hline Single Leg Lunge 1RM (kg) & 14.46 & 29.02 & 38.71 \\
\hline
\end{tabular}

Torque measured in $\mathrm{N} \bullet \mathrm{m}$

Power measured in $\mathrm{W}$ 
Plyometric Group Mean Normative Data

Plyometric Group Mean Normative

\begin{tabular}{|c|c|c|c|}
\hline Variable & Pre & Mid & Post \\
\hline Age & 64.00 & 64.00 & 64.00 \\
\hline Height (cm) & 165.81 & 165.81 & 165.81 \\
\hline Weight (kg) & 71.53 & 71.20 & 70.86 \\
\hline Body_Fat (\%) & 34.54 & & 35.40 \\
\hline BMI $\left(\mathrm{kg} / \mathrm{m}^{2}\right)$ & 25.82 & 25.70 & 25.56 \\
\hline Sit/Stand (s) & 8.04 & 6.76 & 6.30 \\
\hline Stair_Climb (s) & 3.17 & 2.97 & 2.70 \\
\hline $60 \%$ sec Torque Flexion & 74.79 & 82.18 & 82.50 \\
\hline $60^{\circ} / \mathrm{sec}$ Torque Extension & 86.42 & 92.16 & 96.94 \\
\hline $60 \% /$ sec Power Flexion & 52.56 & 57.63 & 57.26 \\
\hline $60 \% /$ sec Power Extension & 59.33 & 60.81 & 64.50 \\
\hline $120 \%$ sec Torque Flexion & 67.08 & 70.35 & 55.20 \\
\hline $120 \%$ sec Torque Extension & 68.11 & 73.20 & 76.16 \\
\hline $120 \%$ sec Power Flexion & 93.80 & 94.05 & 103.56 \\
\hline $120^{\circ} /$ sec Power Extension & 83.50 & 86.58 & 97.76 \\
\hline $180^{\circ} /$ sec Torque Flexion & 61.44 & 63.49 & 66.96 \\
\hline $180^{\circ} /$ sec Torque Extension & 59.88 & 60.79 & 67.91 \\
\hline $180^{\circ} /$ sec Power Flexion & 111.34 & 116.05 & 132.20 \\
\hline $180^{\circ} /$ sec Power Extension & 103.94 & 101.14 & 116.90 \\
\hline Leg Press 1RM (kg) & 143.53 & 153.06 & 155.69 \\
\hline Leg Extension 1RM (kg) & 61.35 & 67.27 & 70.92 \\
\hline Single Leg Lunge 1RM (kg) & 18.76 & 26.83 & 30.65 \\
\hline
\end{tabular}

Torque measured in $\mathrm{N} \bullet \mathrm{m}$

Power measured in $\mathrm{W}$ 


\section{Control Group Mean Normative Data}

Control Group Mean Normative Data

\begin{tabular}{|c|c|c|c|}
\hline Variable & Pre & Mid & Post \\
\hline Age & 62.71 & 62.71 & 62.71 \\
\hline Height (cm) & 166.50 & 166.50 & 166.50 \\
\hline Weight (kg) & 67.96 & 68.54 & 68.97 \\
\hline Body_Fat (\%) & 37.43 & & 39.13 \\
\hline BMI $\left(\mathrm{kg} / \mathrm{m}^{2}\right)$ & 24.53 & 24.75 & 24.89 \\
\hline Sit/Stand (s) & 8.25 & 8.35 & 7.82 \\
\hline Stair_Climb (s) & 3.14 & 3.24 & 3.20 \\
\hline $60^{\circ} /$ sec Torque Flexion & 65.20 & 67.13 & 70.87 \\
\hline $60^{\circ} / \mathrm{sec}$ Torque Extension & 82.14 & 77.94 & 82.12 \\
\hline $60^{\circ} /$ sec Power Flexion & 44.23 & 47.20 & 48.16 \\
\hline $60 \%$ sec Power Extension & 52.00 & 50.13 & 53.51 \\
\hline $120 \% /$ sec Torque Flexion & 56.44 & 62.39 & 45.01 \\
\hline $120 \% / s e c$ Torque Extension & 61.09 & 64.21 & 66.36 \\
\hline $120^{\circ} / \mathrm{sec}$ Power Flexion & 67.11 & 72.06 & 73.34 \\
\hline $120^{\circ} /$ sec Power Extension & 70.51 & 75.27 & 75.84 \\
\hline $180^{\circ} /$ sec Torque Flexion & 53.07 & 60.78 & 55.57 \\
\hline $180^{\circ} / \mathrm{sec}$ Torque Extension & 52.37 & 54.45 & 55.34 \\
\hline $180^{\circ} /$ sec Power Flexion & 84.31 & 87.37 & 86.59 \\
\hline $180^{\circ} /$ sec Power Extension & 80.20 & 80.94 & 81.41 \\
\hline Leg Press 1RM (kg) & 132.62 & 127.04 & 132.32 \\
\hline Leg Extension 1RM (kg) & 52.27 & 55.61 & 55.91 \\
\hline Single Leg Lunge 1RM (kg) & 12.47 & 15.68 & 17.05 \\
\hline
\end{tabular}

Torque measured in $\mathrm{N} \bullet \mathrm{m}$

Power measured in $\mathrm{W}$ 
APPENDIX H

Progression Graph for Leg Press and Squat Jump 


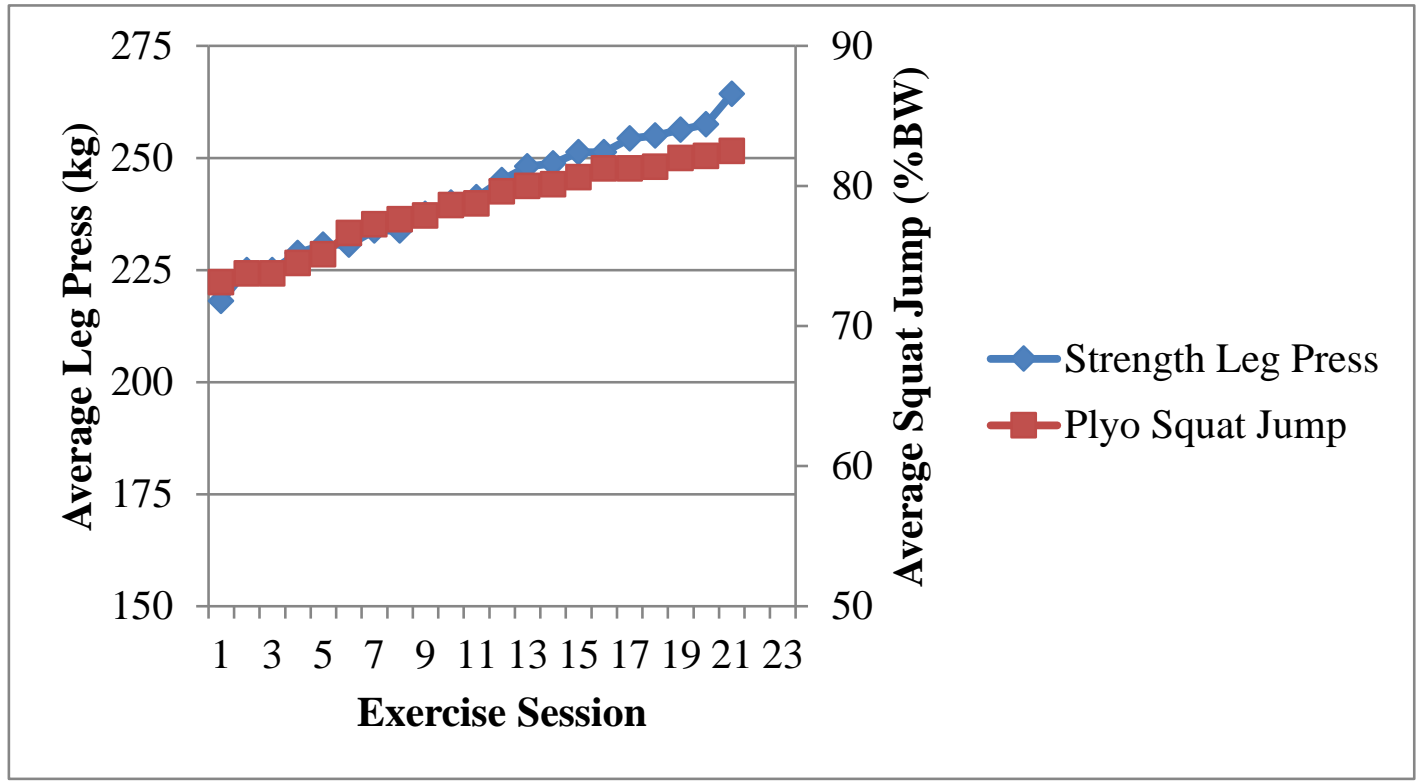

Leg Press and Squat Jump Progression 